PNL-7821

UC-900

Approved for public release; distribution is unlimited

\title{
Microscope-Quantitative Luminescence Imaging System (M-QLIS) Description and User's Manual
}

C. R. Batishko

K. A. Stahl

October 1991

Prepared for

Armstrong Laboratory for Human Systems

Brooks Air Force Base, Texas

under a Related Services Agreement

with the U.S. Department of Energy

Contract DE-AC06-76RLO 1830

Pacific Northwest Laboratory

Operated for the U.S. Department of Energy

by Battelle Memorial Institute 


\title{
DISCLAIMER
}

This report was prepared as an account of work sponsored by an agency of the United States Government. Neither the United States Government nor any agency thereof, nor Battclle Mcmorial Institute, nor any of their employees, makes any warranty, expressed or implied, or assumes any legal liability or responsibility for the accuracy, completeness, or usefulness of any information, apparatus, product, or process disclosed, or represents that its use would not infringe privately owned rights. Reference herein to any specific commercial product, process, or service by trade name, tradernark, manufacturer, or olherwise does not necessarily constitute or imply its endorsement, recommendation, or favoring by the United States Government or any agency thereof, or Battelle Memoriai Institute. The views and opinions of authors expressed herein do not necessarily statc or reflect those of the United Stales Government or any agency thereof.

\author{
PACIFIC NORTHWEST LABORATORY \\ operated by \\ BATTELLE MEMORIAL INSTITUTE \\ for the \\ UNITED STATES DEPARTMENT OF ENERGY \\ under Contract DE-AC06-76RLO 1830
}

Printed in the United States of America

Available to DOE and DOE contractors from the

Office of Scientific and Technical Iníormation, P.O. Box 62, Oak Ridge, TN 371331; prices available írom (615) 576-8401, FIS 626-0401.

Availahle to the public from the National Technical Information Service, U.S. Department of Cummerce, 52115 Port Royal Rd., Springfield, VA 22161. 
MICROSCOPE-QUANTITATIVE LUMINESCENCE IMAGING SYSTEM (M-QLIS) DESCRIPTION ANO USER'S MANUAL

C. R. Bat ishko

K. A. Stahl

October 1991

Prepared for

Armstrong Laboratory for Human Systems Brooks Air Force Base, Texas under a Related Services Agreement with the U.S. Department of Energy

Contract DE-AC06-76RL0 1830

Pacific Northwest Laboratory

Richland, Washington 99352 

A Microscope Quantitative Luminescence Imaging System (M-QLIS) has been designed and constructed. The M-QLIS is designed for use in studies of chemiluminescent phenomena associated with absorption of radio-frequency radiation. The system consists of a radio-frequency waveguide/sample holder, microscope, intensified video camera, radiometric calibration source and optics, and computer-based image processor with radiometric analysis software. The system operation, hardware, software, and radiometric procedures are described. 


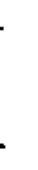




\section{CONTENTS}

SUMMARY

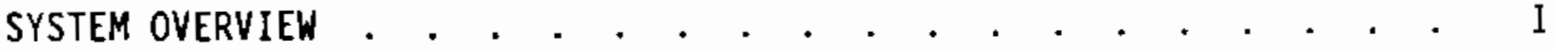

HARDWARE . . . . . . . . . . . . . . . . . . . . 3

MICROSCOPE . . . . . . . . . . . . . . . . . . 3

RF SAMPLE HOLDER . • . . . . . . . . . . . . . . 5

CALIBRATION ASSEMLBY . • . . . . . . . . . . . . . . . . 6

CAMERA . . . . . . . . . . . . . . . . 7

COMPUTER . . . . . . . . . . . . . . . . . . . 7

ASSEMBLY AND OPERATION . . . . . . . . . . . . . . . . . . . . . . . 11

MICROSCOPE ASSEMBLY • . . • . . . . . . • . . . . 11

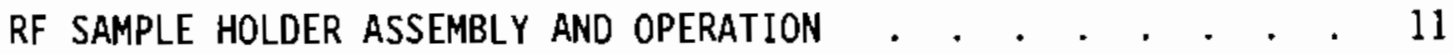

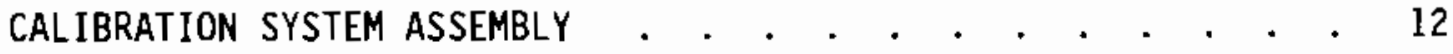

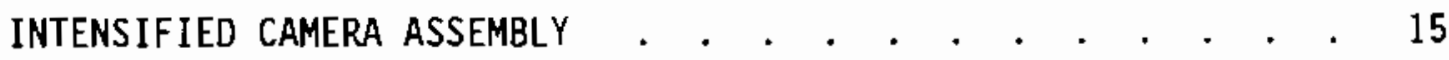

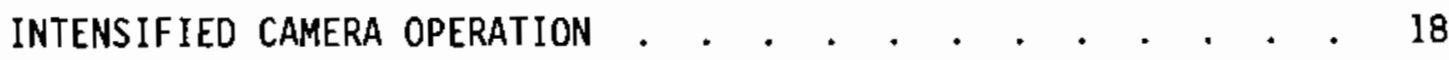

COMPUTER/IMAGE PROCESSOR

SOFTWARE - . . . . . . . . . . . . . . . . . . 21

RADIOMETRIC ANALYSIS PROCEDURES . . . . . . . . . . . . . . . 23

IMAGE ACQUISITION AND PREPARATION PROCEDURE . . . . . . . . 23

RADIOMETRIC ANALYSIS PROCEDURE . . . . . . . . . . . . 30

CALIBRATION . . . . . . . . . . . . . . . . . . . . . . . . 35

PRIMARY CALIBRATION PROCEDURE . . . . . . . . . . . . . 35

CALIBRATION CHECK PROCEDURE . . . . . . . . . . . . . . . 38

RADIANCE CALCULATIONS . . . . . . . . . . . . . . . . . . . . 43

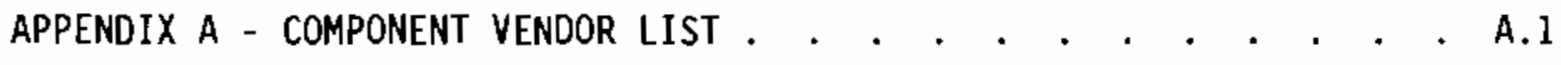

APPENDIX B - RF SAMPLE HOLDER DETAILS . . . . . . . . . . . . . . B.l 


\section{FIGURES}

1. The Complete M-QLIS . . . . . . . . . . . . . . . 3

2. The M-QLIS Microscope, with Calibration Mirror and Reticle Holder . . . . . . . . . . . . . . . . . . 4

3. The RF Sample Holder Installed under the Microscope . . . . 5

4. The Calibration Radiance Source . . . . . . . . . . . 6

5. The Intensified Camera . . . . . . . . . . . . . . 8

6. The M-QLIS Power Supplies . . . . . . . . . . . . . 9

7. Calibration Source and Lens Holder Mounted to the Baseplate. . 12

8. Proper Position of the Reticle atop the Mirror/Reticle Holder . 14

9. Proper Alignment of the Calibration Assembly and the Microscope Base . . . . . . . . . . . . . . . . 15

10. The Cross-hair Insert Installed in the Calibration Radiance Source Output Port . . . . . . . . . . . . . . . 16

11. Reticle and Cross-hair Pattern as Viewed through the Microscope. 17

12. The Aperture and Filter Installed in the Calibration Radiance Source Output Port . . . . . . . . . . . . . . . 18

13. The IMAge-PRO Main Menu . . . . . . . . . . . . . . 22

14. The File Sub-Menu . . . . . . . . . . . . . . . . 24

15. The Acquire Sub-Menu . . . . . . . . . . . . . . . 24

16. The Save Image Directory. . . . . . . . . . . . . . 25

17. The Select Area Sub-Menu. . . . . . . . . . . . . . 25

18. The Options Sub-Menu . . . . . . . . . . . . . . . 27

19. The Select AOI Image Sub-Menu . . . . . . . . . . . . 27

20. The Select a01 Sub-Menu . . . . . . . . . . . . . . 28

21. The Box Sub-Menu . . . . . . . . . . . . . . 28

22. The User Modules Sub-Menu . . . . . . . . . . . . . 29 


\section{FIGURES (continued)}

23. The Analysis Sub-Menu . . . . . . . . . . . . . . 31

24. The Histogram Screen . . . . . . . . . . . . . . . 31

25. The Histogram Screen, Showing the Display accumulated? Prompt . 32

26. The Histogram Screen, Showing the Calculate Range of Indexes?

Prompt . . . . . . . . . . . . . . . . 32

27. The Save Data Directory . . . . . . . . . . . . . 33

28. The User Modules Sub-Menu, Showing Selection of the Radiometry User Module. . . . . . . . . . . . . . . . . . 33

29. Primary Calibration Curves for the $2.5 x$ Objective . . . . . 37 


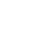




\section{SYSTEM OVERVIEW}

The M-QLIS was developed for use in imaging and quantitative analysis of very low light level chemiluminescent phenomena taking place on the microscopic level. The chemiluminescence is imaged via a conventional microscope coupled to an intensified video camera, which have been radiometrically calibrated in order to relate video signal levels to true radiometric quantities. The video data can be captured (digitized) and analyzed with the system's microcomputer-based frame grabber/image processor hardware and software. Through the use of a radiometric calibration procedure, radiometric analysis software converts the digitized video grey levels to the photon flux (in units of photons/second) being produced by the sample.

Since the research program for which the M-QLIS was developed is aimed at investigating phenomena associated with the absorption of radio-frequency (RF) radiation, an $\mathrm{RF}$ waveguide/sample holder was developed for the M-QLIS. This waveguide/sample holder can be used to deliver RF radiation to the sample and to position the sample under the microscope. The modular design of the microscope itself allows for a high degree of flexibility in implementation of additional or modified sample holders, etc. 


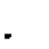




\section{HARDWARE}

This section describes the M-QLIS hardware. The complete system is pictured in Figure 1. It is convenient to consider the hardware in four major sub-systems: the microscope, the calibration source, the RF sample holder, and the computer. More detailed information on the various commercial equipment comprising the M-QLIS is available in the corresponding manual(s) provided by the equipment vendors. A vendor list appears in Appendix A.

\section{MICROSCOPE}

The M-QLIS microscope, pictured in Figure 2, is an 0lympus model \#BHMJ. This model offers a post-mounted modular design which lends itself very well to the modifications required for the M-QLIS, both present and future. The

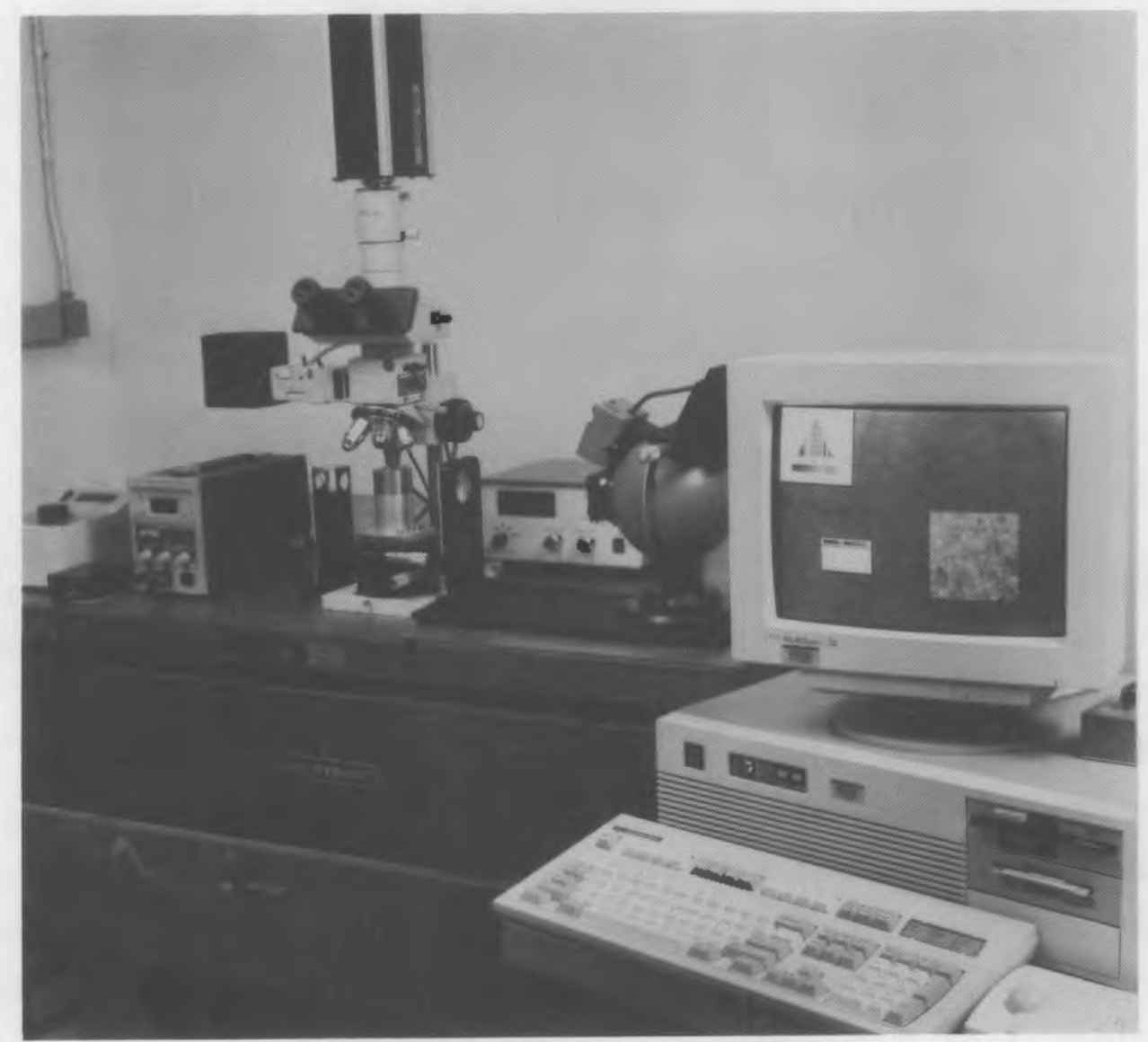

FIGURE 1. The Complete M-QLIS 


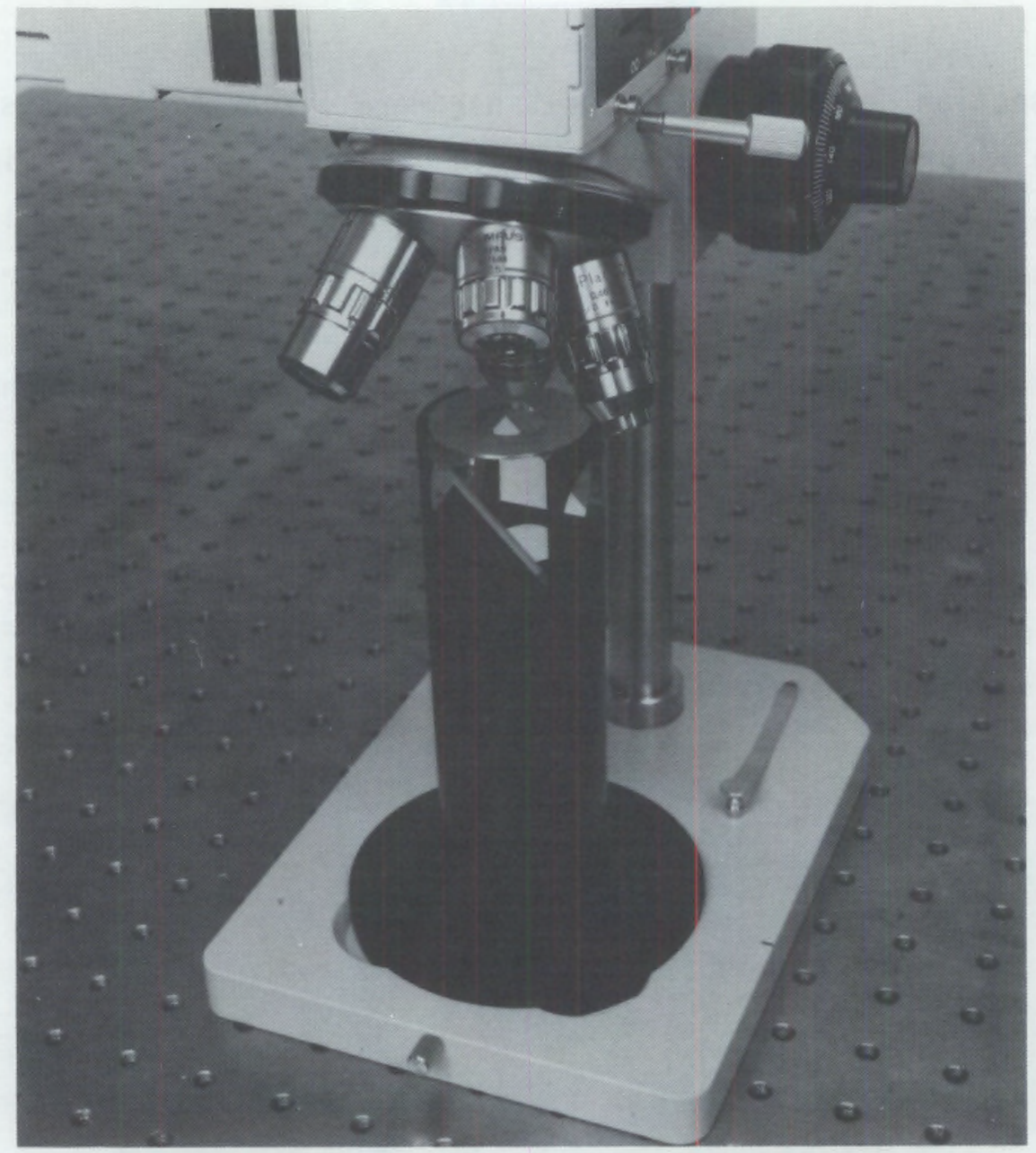

FIGURE 2. The M-QLIS Microscope, with Calibration Mirror and Reticle Holder

microscope features a trinocular observation tube with a video camera adapter (\#MTV-3) and a universal vertical illuminator with a bright field beam splitter cube, halogen lamp housing, and power supply. The vertical illuminator is not used during normal M-QLIS radiometric operations, but is available for non-quantitative observations. The objectives provided with the system are MS Plan Achromats, with powers of 2.5X, 5X, 10X, 20X, and 50X. The microscope does not include a standard sample stage (with $X-Y$ micrometer adjustment, etc.). Instead, $X-Y$ translation stages are included on the RF sample holder, providing sample positioning capability comparable to a standard sample stage. The microscope is used in the "stock" configuration, 
except for the mounting post, which has been replaced with a longer post fabricated at PNL.

\section{RF SAMPLE HOLDER}

The RF sample holder, shown in Figure 3, provides a means of delivering an RF field to the sample, while still allowing the sample to be positioned and viewed under the microscope. The unit is designed to provide an optimal uniform field at a frequency of $2.45 \mathrm{GHz}$. RF excitation is applied to the sample holder via an SMA co-axial connector. The RF excitation is delivered to a recessed culture dish holder which is sized for $35 \mathrm{~mm} \times 10 \mathrm{~mm}$ disposable dishes, such as the Corning \#25000-35. The RF portion of the assembly is

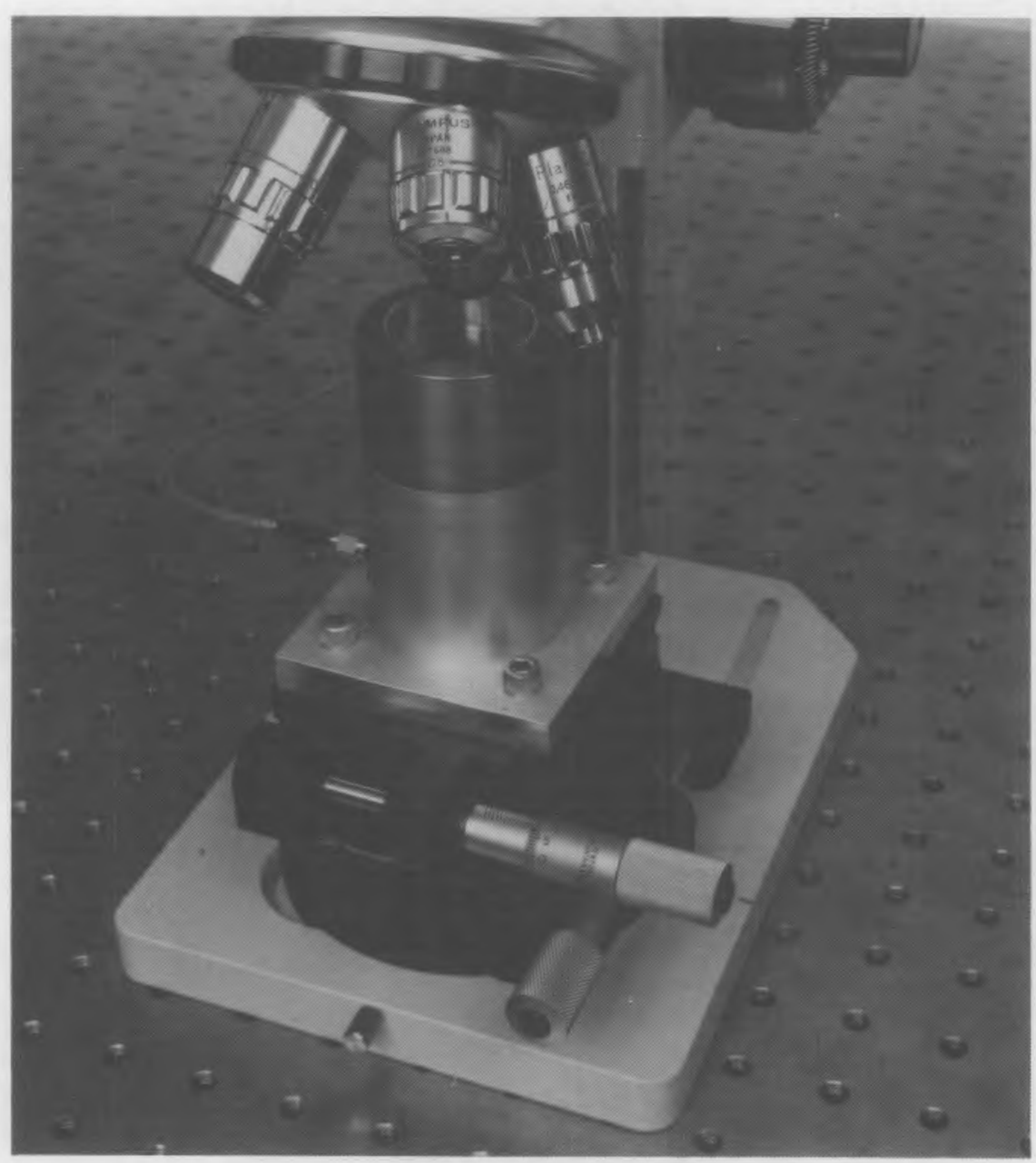

FIGURE 3. The RF Sample Holder Installed under the Microscope 
mounted on $X-Y$ translation stages with micrometer positioners. The translation stages are, in turn, attached to a stage plate which fits in the microscope's stage plate opening. Additional detail on the RF sample holder is given in Appendix B.

\section{CALIBRATION ASSEMBLY}

The calibration source, pictured in Figure 4, is a Hoffman Engineering model \#RS-65-C Spectral Radiance Standard. The source provides NISTtraceable spectral radiance which is used to derive the primary calibration for the M-QLIS. The source uses a thermally stabilized tungsten lamp in an integrating sphere, which provides a spatially uniform radiance output. The radiance level can be adjusted over approximately 5 orders of magnitude via a micrometer-controlled variable slit between the tungsten source and the

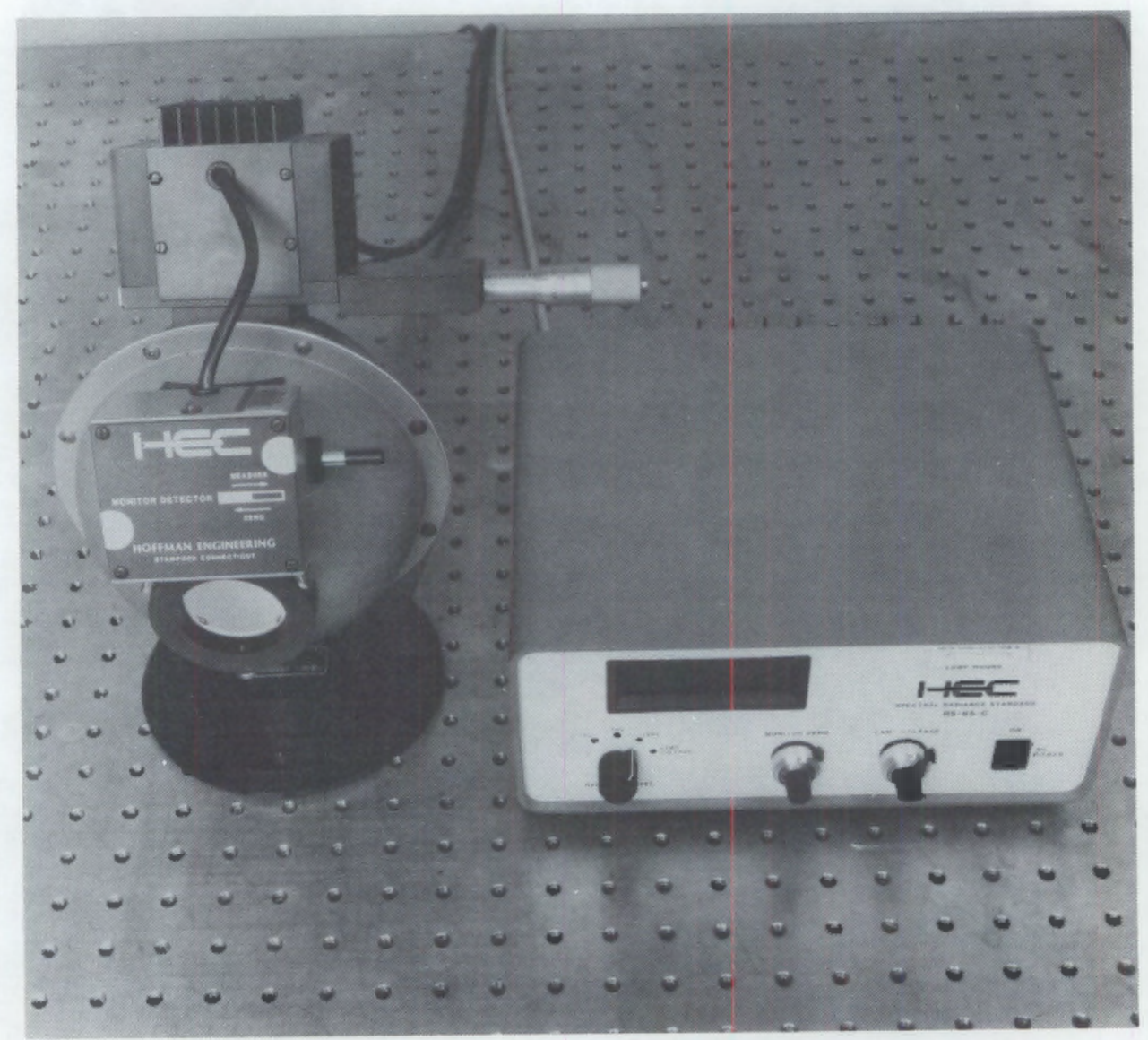

FIGURE 4. The Calibration Radiance Source 
integrating sphere. The source controller provides an elapsed time indicator which keeps track of the total operation time of the source, facilitating recalibration of the source at the proper intervals (manufacturer recommends recalibration yearly or after 100 hours of use). The source is mounted to a base plate, upon which is mounted a focusing lens. The purpose of the focusing lens, in combination with a beam-steering mirror and focusing reticle, is to focus an image of the calibration source output aperture onto the object plane of the microscope, thereby placing a known radiance in the object plane. A front-surface aluminum mirror is mounted, along with a focusing reticle, in a holder which fits into the microscope's stage plate opening. The holder is designed so that the mirror is held at 45 degrees with respect to the output plane of the calibration source, so that the converging cone of light from the focusing lens arrives at the object plane of the microscope. The calibration source and focusing lens are arranged so that the image of the calibration source output aperture is coincident with the focusing reticle. An alignment notch on the top of the base plate corresponds to a notch on the base of the microscope.

\section{CAMERA}

The M-QLIS camera, shown in Figure 5 , is an ITT model \#F4577. This camera features an intensified CCD array and provides standard RS-170 video output. The intensifier gain can be controlled by a precision 10-turn potentiometer on the camera case, or externally via a $0-9.7$ volt $D C$ signal. The camera has a C-mount lens flange which is compatible with the microscope's video camera adapter, allowing the camera to be securely fastened to the microscope's camera tube. The camera is powered by an externa1 15 volt DC power supply. An additional DC power supply is provided with the M-QLIS. This power supply provides the operator with a simple means of applying the variable $D C$ voltage required for external gain control. The two power supplies are pictured in Figure 6.

\section{COMPUTER}

The M-QLIS computer is a Gateway 2000 IBM AT-compatible with $25 \mathrm{MHz}$ 80386 microprocessor; 140 MB hard disk; 1.2 MB 5.25" and 1.4 MB 3.5" floppy 


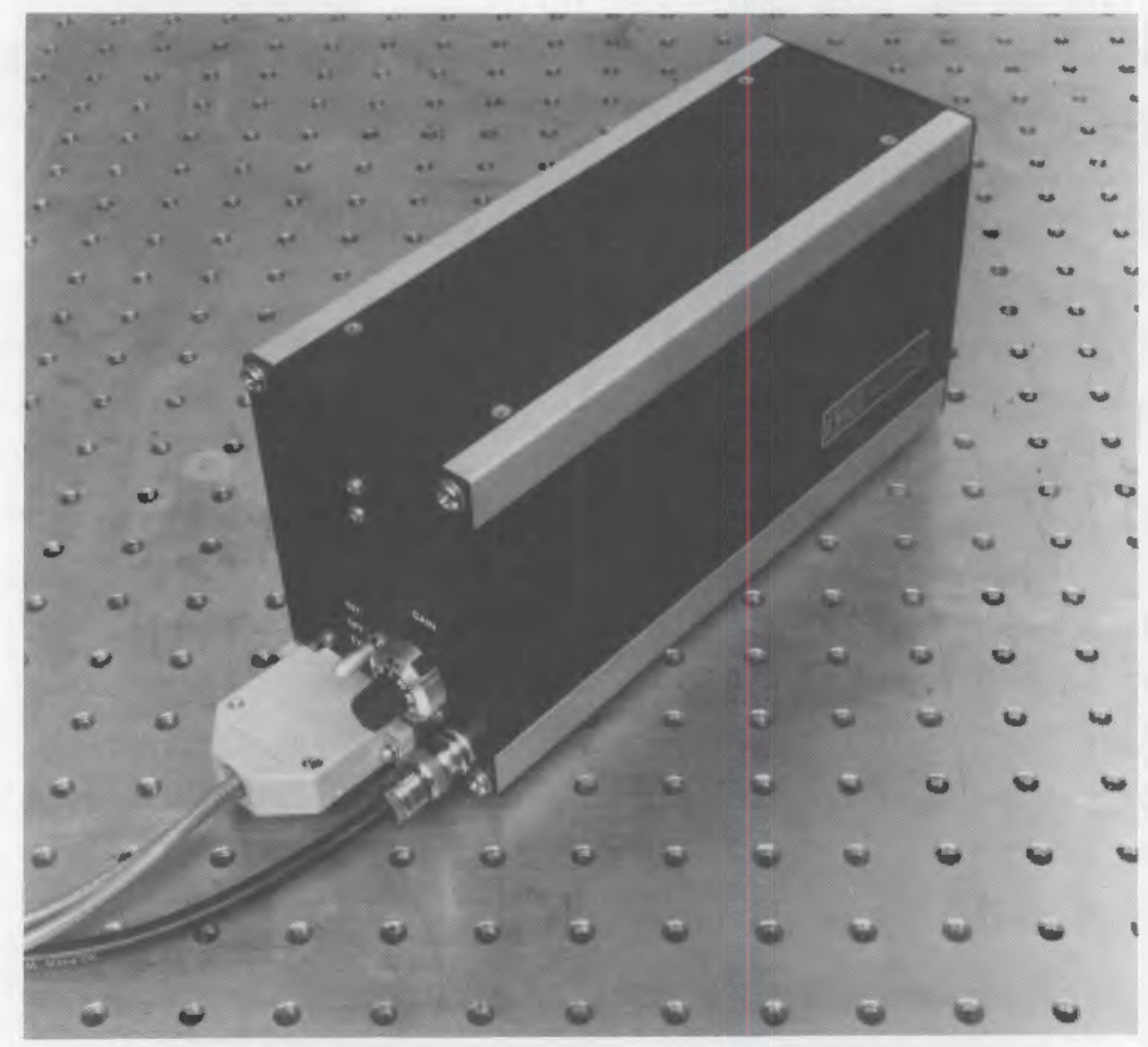

FIGURE 5. The Intensified Camera

drives; Alphatronix erasable optical disk drive; VGA graphics card; multisync color monitor; mouse; and an Imaging Technology VS-100/640 frame grabber/ processor board. The computer uses the DOS 4.01 operating system. Software provided with the M-QLIS computer includes Image Pro 2.01 (image processing software supporting the VS-100/640, produced by Media Cybernetics, Inc.), and Microsoft QuickBASIC 4.5.

The VS-100/640 frame grabber/processor plugs into a standard expansion slot in the computer. This board offers $640 \times 480$ pixel resolution and can capture images in standard RS-170 video format or provide the necessary clocking signals to capture non-standard format images, such as those from slow-scan cameras. The board provides color (RGB) and black and white (RS-170) video output. A switch box allows the operator to toggle the 


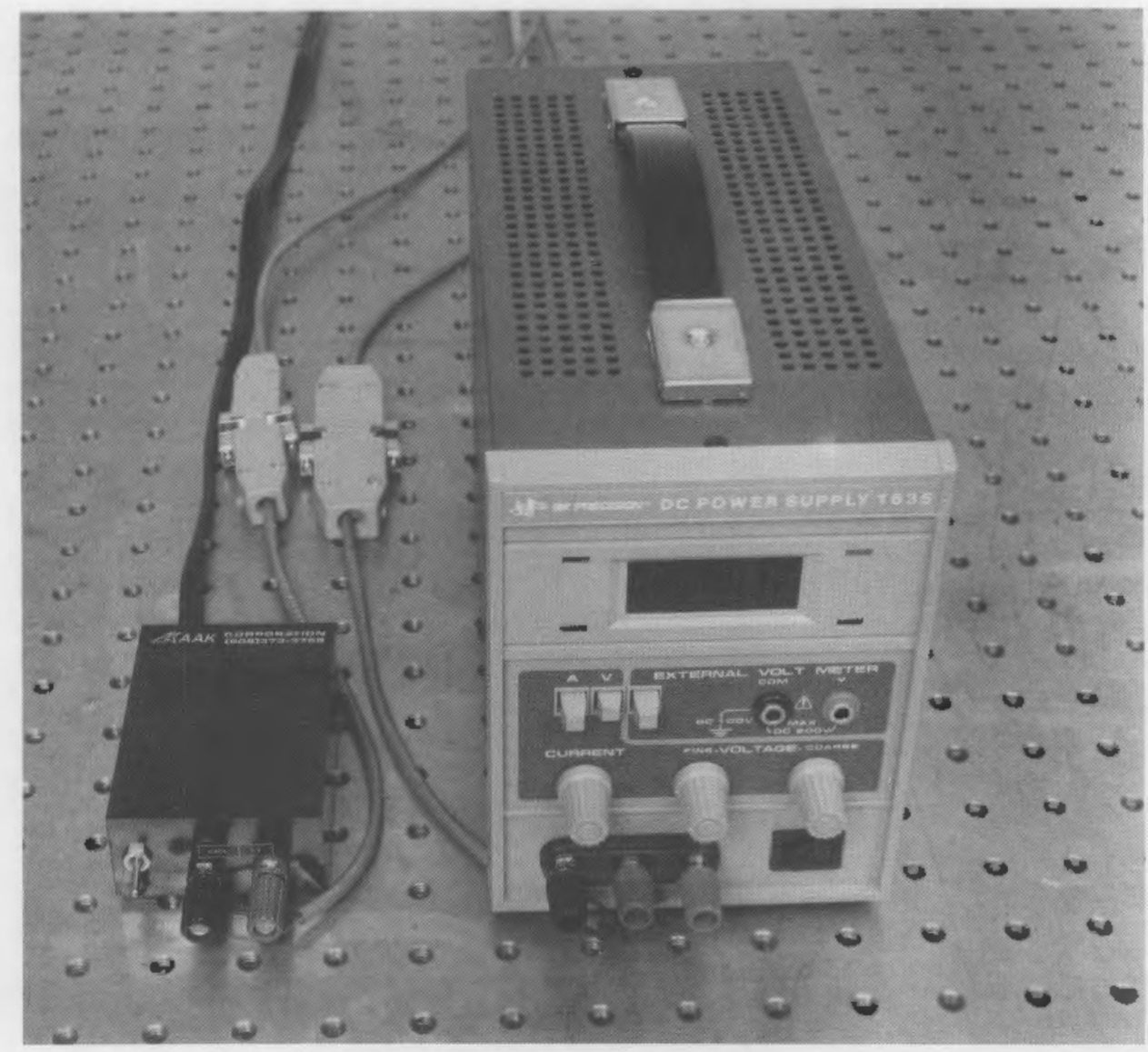

FIGURE 6. The M-QLIS Power Supplies for the Intensified Camera (left) and External Gain Control (right)

computer's multisync color monitor between display of computer output and display of image processor output. 


\section{ASSEMBLY AND OPERATION}

The assembly of the M-QLIS is very straightforward, since the system is modularized to a high degree. No practice or special skills are required. This section provides instructions pertaining to proper assembly and set up of the M-QLIS, as well as basic operational guidelines which are useful for initial check-out of the system and familiarization with its operation.

\section{MICROSCOPE ASSEMBLY}

To assemble the microscope, follow these steps (refer to the microscope vendor manual for detailed assembly instructions):

1. Use three 8-32 machine screws to attach the mounting post to the microscope base.

2. Slide the microscope body onto the mounting post.

3. With the microscope body approximately 8 inches above the baseplate, secure the body by tightening the knob at the back of the body. Further adjustment of the height of the microscope body on the mounting post will be completed after assembly of the calibration system.

4. Screw the microscope objectives into the nose-piece.

5. Attach the trinocular observation tube to the vertical illuminator.

6. Attach the MTV-3 camera adapter to the trinocular tube and align the red dots.

7. If desired, attach the tungsten lamp housing to the illuminator, and connect the lamp cable to the transformer.

\section{RF SAMPLE HOLDER ASSEMBLY AND OPERATION}

Assembly and operation of the RF sample holder is very straightforward:

1. Insert the RF sample holder into the microscope's stage plate opening (TAKE CARE TO AVOID BUMPING THE OBJECTIVES WITH THE SAMPLE HOLDER).

2. Rotate the holder so the notch in the base plate lines up with the stage plate clamping screw. Tighten the screw. 
The RF sample holder is operated by connecting the desired RF power supply to the sample holder with a co-axial cable terminated with SMA connectors. No adjustments, other than the $X-Y$ translation stage are required.

\section{CALIBRATION SYSTEM ASSEMBLY}

The proper arrangement of the calibration source, lens mount, and baseplate is shown in Figure 7. Attach the calibration source to the baseplate using two 1/4-20 flat-head machine screws. The screws should be inserted from the underside of the plate, through the hole furthest from the alignment notch on the left edge of the baseplate, and the corresponding hole 5 inches forward. The calibration source output aperture should face the

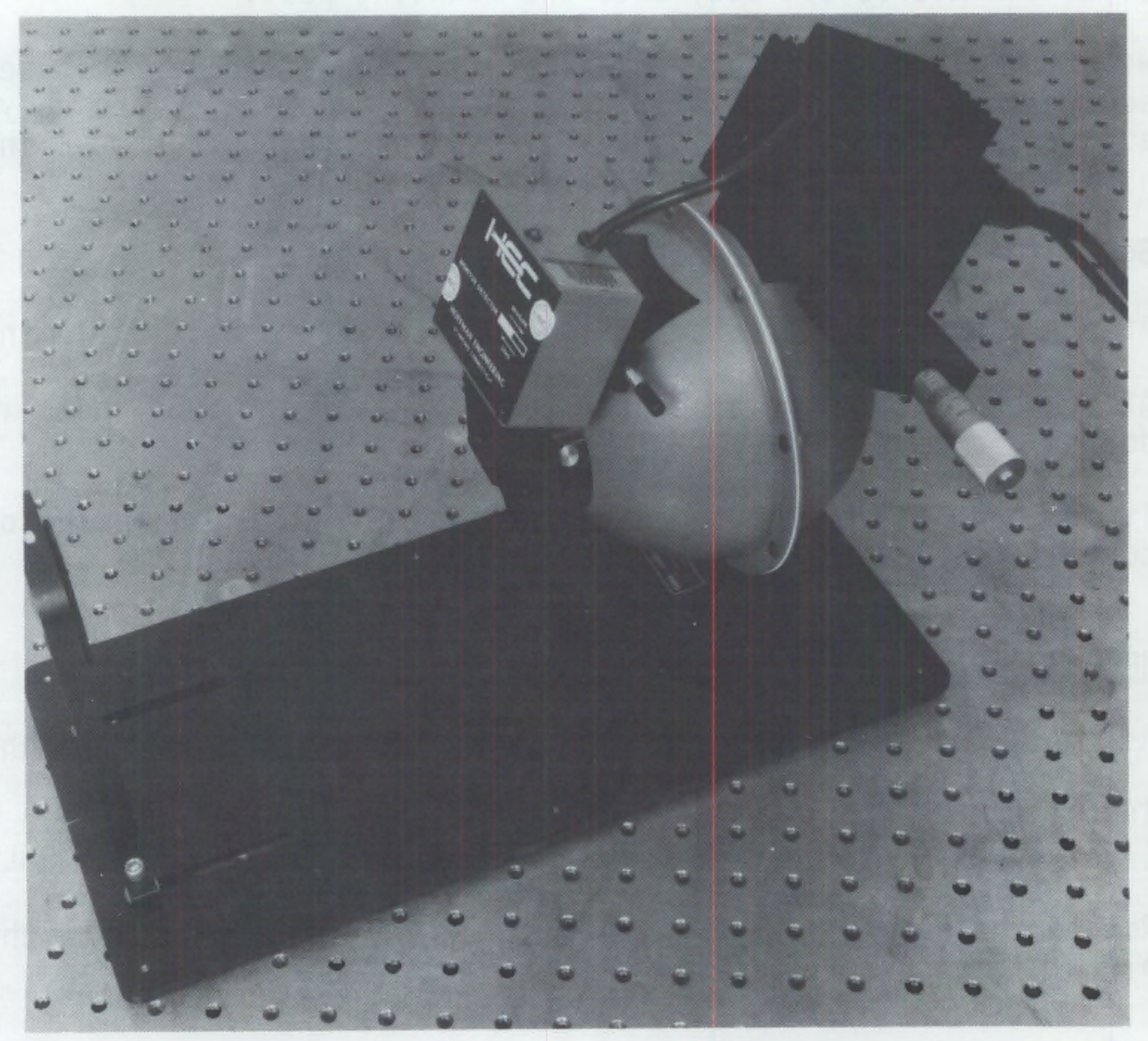

FIGURE 7. Calibration Source and Lens Holder Mounted to the Baseplate 
alignment notch. Attach the lens mount to the baseplate using 1/4-20 machine screws and nuts. The machine screws should be inserted from the top side of the plate, with the nuts resting in the channels machined into the underside of the plate. Do not firmly tighten the two screws at this time.

Insert the focusing lens into the lens mount. The shoulder around the lens holder should face the calibration source. Tighten the 8-32 set screw in the top of the lens mount. Do not over-tighten the set screw--it need only be tightened enough to apply light pressure to the lens holder. Take precautions to avoid marring the surfaces of the lens with fingerprints, etc. If the lens does become smudged, it may be cleaned with lens tissue. The calibration source, lens mount, and baseplate assembly should appear as shown in Figure 7.

Remove the protective cap from the calibration mirror/reticle holder. Being careful to avoid bumping the microscope objectives, place the holder assembly into the microscope stage plate opening, rotate it so the notch in the assembly's base plate lines up with the stage plate clamping screw, and tighten the screw. Place the focusing reticle, with the tapered face down, onto the top of the assembly, and center the reticle by sliding it back toward the microscope mounting post as far as it will go, as shown in Figure 8.

The calibration system and microscope can now be adjusted for proper use. Slide the calibration assembly baseplate so it is flush with the microscope base, with the alignment notches lined up, as shown in Figure 9. Rotate the microscope nose-piece so that the $5 \mathrm{X}$ objective is aligned. With the microscope's focusing block adjusted to approximately the middle of its travel range, partially loosen the knob at the back of the microscope body, and slide the microscope slowly down the mounting post, using a slight sideto-side motion, until the focusing reticle begins to come into view through the microscope eyepieces (be sure that the light path selector on the side of the trinocular tube is set to "V"--pushed all the way in). Securely tighten the knob at the back of the microscope body. Adjust the microscope focus so the reticle is in focus. Note that the orientation of the reticle is not important. Slide the calibration source base plate against the microscope base so that the alignment marks on each are aligned. 


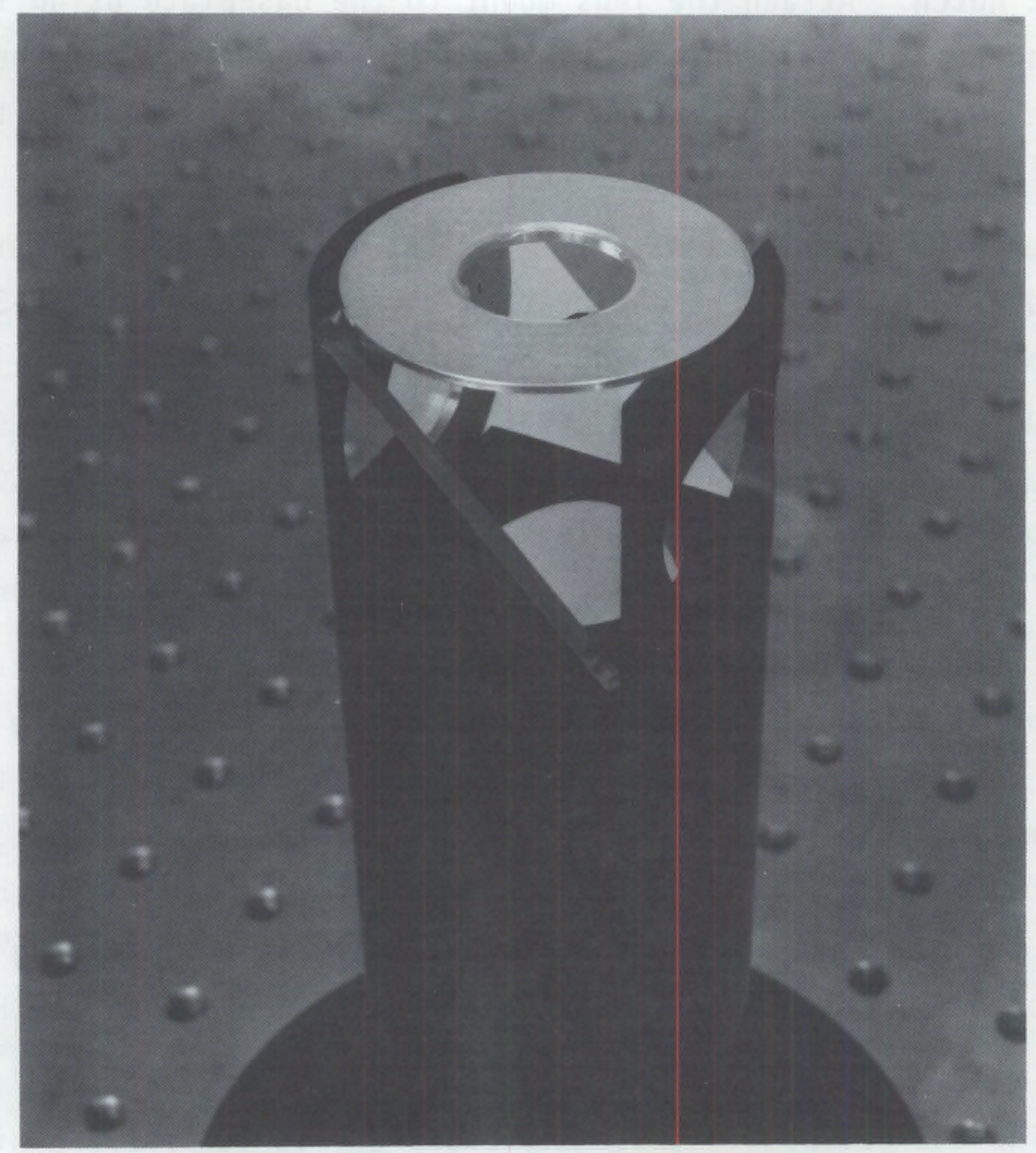

FIGURE 8. Proper Position of the Reticle atop the Mirror/Reticle Holder

Place the cross-hair insert in the calibration source output port, as shown in Figure 10, and tighten the three thumbscrews on the port adapter. Switch on the calibration source power supply, set the range select knob to $100 \%$, and adjust the calibration source micrometer so the source controller's digital display reads at least $20 \%$, but no more than $100 \%$. Rotate the microscope nose-piece to select the $2.5 X$ objective, and adjust the focus, if necessary, to give a sharp image of the reticle. While looking through the microscope, slide the focusing lens mount as necessary to bring the crosshair into focus. Do not adjust the focus of the microscope; when properly adjusted, the reticle and the cross-hair will both be in sharp focus simultaneously, and the cross-hair pattern will be approximately centered in 


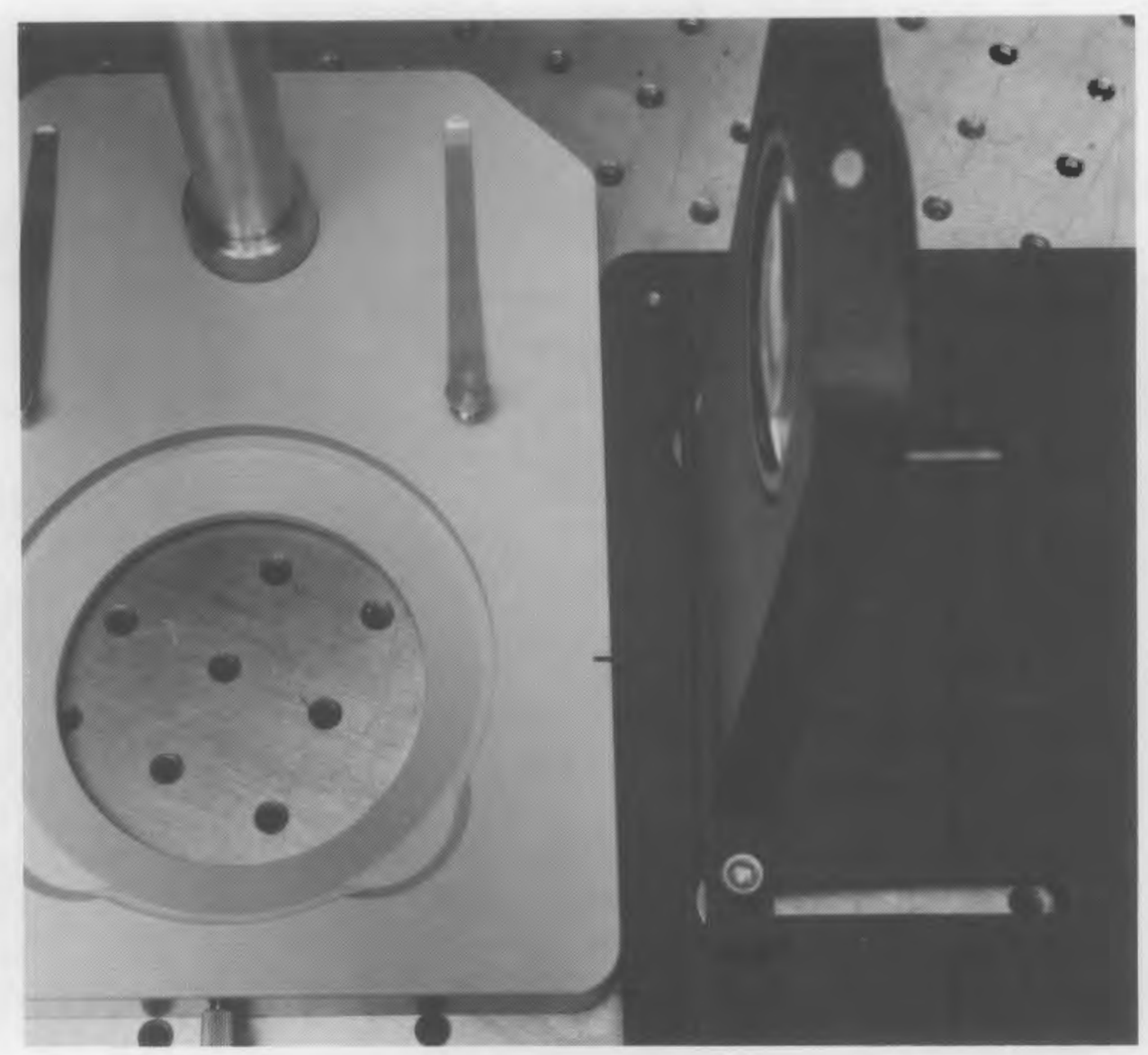

FIGURE 9. Proper Alignment of the Calibration Assembly and the Microscope Base

the field of view, as pictured in Figure 11. Tighten the two screws at the base of the focusing lens mount. If the cross-hair pattern cannot be accurately centered by positioning the lens mount, loosen (slightly) the knob at the back of the microscope body, and swing the microscope body left or right as required to $\mathrm{place}$ the cross-hair at the approximate center of the field of view, then re-tighten the knob at the back of the microscope body.

\section{INTENSIFIED CAMERA ASSEMBLY}

BEFORE OPERATING OR HANDLING THE INTENSIFIED CAMERA, READ AND UNDERSTAND THE CAMERA'S OPERATIDNS MANUAL. Improper use or handling can result in destruction of the camera. Of particular concern is the level of light incident on the camera's faceplate. THE CAMERA SHOULD UNDER NO CIRCUMSTANCES 


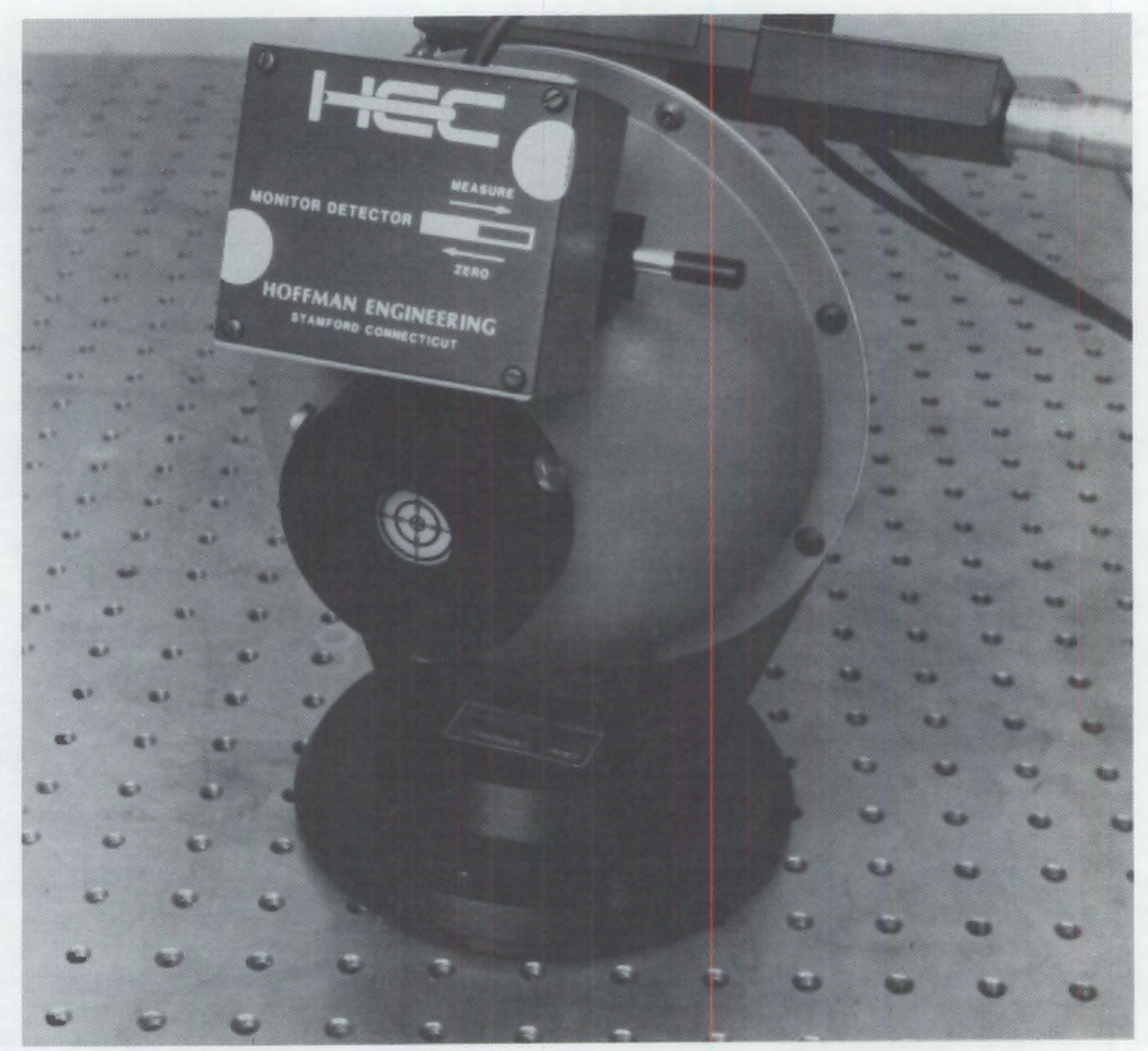

FIGURE 10. The Cross-hair Insert Installed in the Calibration Radiance Source Output Port

\section{BE OPERATED WITHOUT SIMULTANEOUSLY MONITORING ITS VIDEO OUTPUT FOR OVER-} EXPOSURE CONDITIONS. The manufacturer specifies a maximum faceplate illuminance of $1 \times 10^{-4}$ foot-candles, which is approximately equal to the illuminance found in a nearly dark room.

The camera itself requires no assembly. However, the camera must be properly mated to the microscope, and the proper electrical connections must be made. To mount the camera to the microscope, first make sure that the camera is either disconnected from its power supply or switched off. Loosen the set screw which holds the C-mount transfer lens in the microscope's MTV-3 adapter, and remove the $\mathrm{C}$-mount transfer lens. Screw the $\mathrm{C}$-mount lens fully into the camera. When the lens is screwed in tightly, the shoulder of the lens should be snug against the camera's lens adaptor ring. If it is not, 


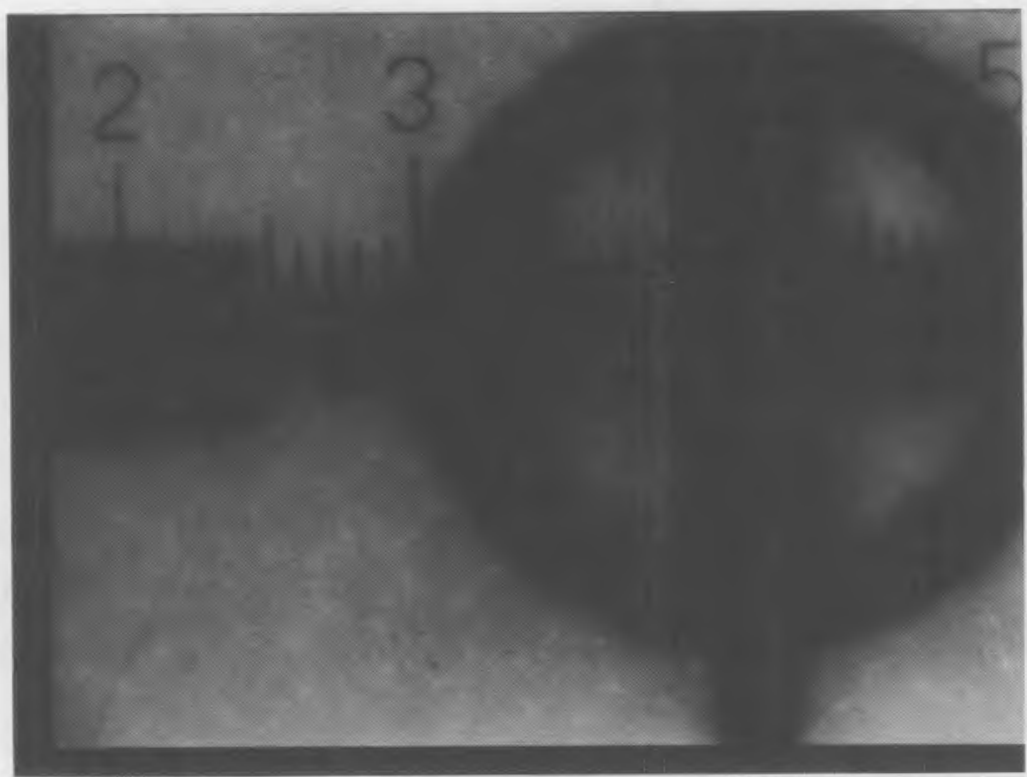

FIGURE 11. Reticle and Cross-hair Pattern as Viewed through the Microscope

loosen the setscrews located in the camera's lens adaptor ring and slide the adaptor ring, with the C-mount transfer lens, as far back into the camera housing as possible, and re-tighten the setscrews. This is required in order to achieve nominal parfocality between the ocular viewing path and the camera viewing path. Slip the $\mathrm{C}$-mount transfer lens, with camera attached, back into the MTV-3, and rotate the transfer lens so the camera housing is square with the microscope (the 1/4-20 mounting holes in the bottom of the camera case should be to the back of the microscope).

Before making any electrical connections, make sure the camera and all power supplies are turned off. If not already connected, connect the 15 volt DC camera power supply to the camera power cable by mating the 9-pin "D" connectors. If desired, also connect the $0-9.7$ volt DC external gain control power supply (switched off) to the camera power cable by mating the 15-pin "D" connectors. Connect the 25-pin "D" connector of the camera power cable to the rear of the camera. 


\section{INTENSIFIED CAMERA OPERATION}

Read and become familiar with the camera's operations manual provided by the vendor. Before applying power to the camera, connect the camera to a suitable video monitor with $75 \mathrm{ohm}$ co-axial cable. To assure a safe light level and proper operating conditions for initial operation of the camera, the M-QLIS calibration system can be used. Set up the calibration system as described in the previous section of this manual. If installed, remove the calibration source's cross-hair insert and replace it with the filter/aperture holder containing the 3/4" aperture and the photometric filter, as pictured in Figure 12. Turn the calibration source on, set the range selector switch to $100 \%$, and adjust the source micrometer for a source controller reading of $40 \%$ to $50 \%$. Rotate the $2.5 X$ objective into position.

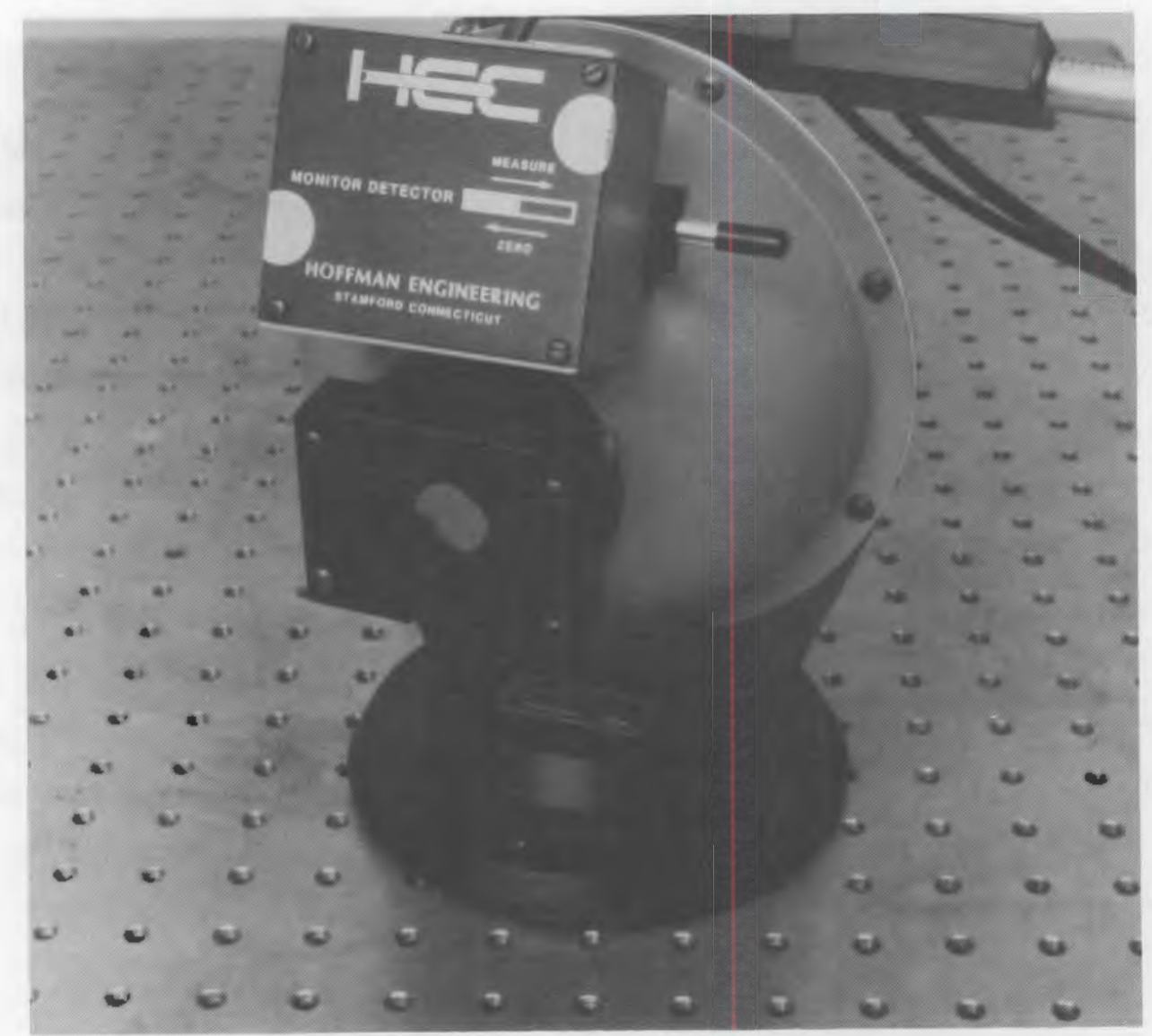

FIGURE 12. The Aperture and Filter Installed in the Calibration Radiance Source Output Port 
Set the INTERNAL-OFF-EXTERNAL switch at the back of the camera to the off position. Set the manual gain setting potentiometer to 0.0 . Switch on the 15 volt $D C$ power supply, and set the camera's switch to INTERNAL. At this point, the intensified camera is running at minimum gain. If the microscope's light path selector is set to either "C.V" (camera and viewer) or "C" (camera only), an image (consisting primarily of the intensifier's fixed-pattern noise) will be on the video monitor; otherwise, the screen will be blank. If the image of the reticle is not in sharp focus, adjust the focus of the microscope as needed. The camera gain may be increased by turning the tenturn potentiometer on the back of the camera. ALWAYS MONITOR THE CAMERA OUTPUT SO THAT OVEREXPOSURE CONDITIONS ARE AVOIDED.

To operate the camera in EXTERNAL gain control mode, first set the INTERNAL gain control potentiometer to 0.0 (to avoid accidentally switching to a high gain condition when going from EXTERNAL to INTERNAL mode). Switch the camera to OFF. Turn the 0-30 volt DC power supply on and set for 0 volts. Switch the camera to EXTERNAL mode. The camera gain may now be adjusted by increasing the $D C$ voltage over the range of 0 to 9.7 volts. DO NOT EXCEED 9.7 VOLTS IN THE* EXTERNAL GAIN MODE. The slope of the gain versus voltage relationship is very steep, so even a slight over-voltage causes a large increase in gain. Always return the power supply to 0 volts before turning the camera off or switching to INTERNAL mode.

The possibility of applying more than 9.7 volts in EXTERNAL mode may be minimized by using the current limit feature of the $0-30$ volt power supply. A 9.1 volt zener diode has been inserted across the voltage pins inside the 25pin "D" connector at the rear of the camera. The zener diode assures that appreciable current is being drawn from the power supply when the supply is set for greater than 9.1 volts. To set the current limit so the power supply cannot provide more than 9.7 volts, first push in the microscope's light path selector, so no light reaches the camera. Next, with the camera in EXTERNAL gain mode, carefully set the power supply for 9.7 volts. Turn the current limit knob clockwise until the power supply just switches to current limiting operation. When the power supply enters the current limiting mode, an audible "click" can be heard, and the power supply's red "C.C." (current control) indicator will light. If the knob is turned further clockwise, the output 
voltage will drop below 9.7 volts. Re-adjust the current limit knob so the power supply reads 9.7 volts. In future operation, this current limiting setting will help assure that no voltage greater than 9.7 volts is applied.

\section{COMPUTER/IMAGE PROCESSOR}

Attach the 9-pin "D" mouse connector to the 9-pin outlet labeled "COMl" at the rear of the computer. Attach the ribbon cable with the two software "hard keys" to the printer port at the rear of the computer, directly underneath the mouse connector. If a system printer is being installed, attach its cable to the last hard key--the keys are transparent to the printer.

Attach one end of the 15-pin "D" connector cable to the corresponding receptacle on the rear of the computer. Attach the other end of this cable to the video switch box, at the receptacle labeled "VGA". Attach the cable from the NEC monitor to the video switch box, at the "OUT" receptacle. Attach the 25-pin end of the image processor cable (the cable with a 25-pin "D" connector on one end, and 13 BNC connectors on the other) to the remaining 25-pin receptacle at the rear of the computer. Attach the three BNC connectors labeled "Red", "Green", and "Blue" to the video switch box receptacles labeled "R", "G", and "B", respectively. Note that the "H" and "V" receptacles are not normally required with the NEC monitor. Attach the BNC connector labeled Cam 1 to the intensified camera, using 75 ohm co-axial cable. A11 other BNC connectors of the image processor cable have no connection.

If the video switch box is to be omitted, connect the cable from the NEC monitor directly to the 15-pin "D" receptacle at the rear of the computer. Attach the "Green" BNC connector from the image processor cable to suitable RS-170 compatible monitor. With the exception of the "Cam 1" connector, all other BNC connectors of the image processor cable have no connection.

If installed, set the video switch box to display "VGA." Turn on the computer and monitor(s). After the computer boots up, the DOS $C: \backslash$ prompt will be displayed. At this time, if the video switch box has been omitted, the RS-170 monitor will be displaying the output of the image processor, which will likely be a blank screen, since the image processing hardware will not 
yet have been initialized. If the switch box is installed, the image processor output can be displayed on the computer's main monitor by setting the box's switch to "RGB". Practical experience has shown that the NEC monitor occasionally fails to correctly lock to the RGB input, so it may be necessary to momentarily switch to VGA and then back to RGB in order to establish the correct sync.

\section{SOFTWARE}

The M-QLIS software consists of the image processing software, IMAGEPRO II, from Media Cybernetics, Inc., and additional software for radiometric analysis of images captured with the frame grabber/processor. IMAGE-PRO is a high-level, menu-driven package that uses the frame grabber/processor's capabilities to grab images, apply contrast manipulations and filters, pseudocolorize images, save and restore images, and so on. IMAGE-PRO is organized as a main program module, through which most of the image processing functions are accessed, and a set of "User's Modules." The user's modules offer additional functions which make use of the special features of the particular frame grabber/processor being used, and allow user-written sub-programs to be run from the Image Pro environment.

The user-written modules are programmed in Microsoft quickBASIC. These modules include Flatten image, which applies correction factors to an image so that spatial gain non-uniformity of the camera can be accounted for; Radiometry, which calculates the number of photons per second being emitted by the image area of interest; and Calib. check, which stores the periodic calibration check value in the proper format for subsequent use by the Radiometry module.

Two programs are provided in addition to the software routines which are accessed from within IMAGE-PRO. CURVTABL is run from the DOS prompt and is used to enter spectral data, such as new sample spectral content curves, camera spectral response curves, etc. MAKFCTRS is also run from the DOS prompt and is used to calculate a file which contains the spatial correction factors that are used by the Flatten image user's module. Under normal circumstances, there will be no need to run MAKFCTRS. 
Since full instructions for operation of IMAGE-PRO are presented in the IMAGE-PRO user's manual, discussion of IMAGE-PRO's operation wil1 be 1 imited to that which is necessary to explain the procedures for using the quantitative radiometric capabilities of the M-QLIS.

To run IMAGE-PRO, first use the cd $\backslash$ imagepro command to enter the Image Pro sub-directory. The $C: \backslash I M A G E P R 0\rangle$ prompt will be displayed. At the prompt, type imagepro 〈Return〉. Alternatively, simply type ip 〈Return〉 from the $\mathrm{C}: 1$ prompt. The latter method utilizes batch file which automatically executes the series of statements used in the first method and returns the user to the C: $\backslash$ prompt when IMAGE-PRO is exited. Upon entering IMAGE-PRO, the main menu will be displayed on the image processor monitor (RS-170 monitor or computer monitor), as shown in Figure 13, and the various functions will be accessible by pointing and clicking with the mouse/trackball.

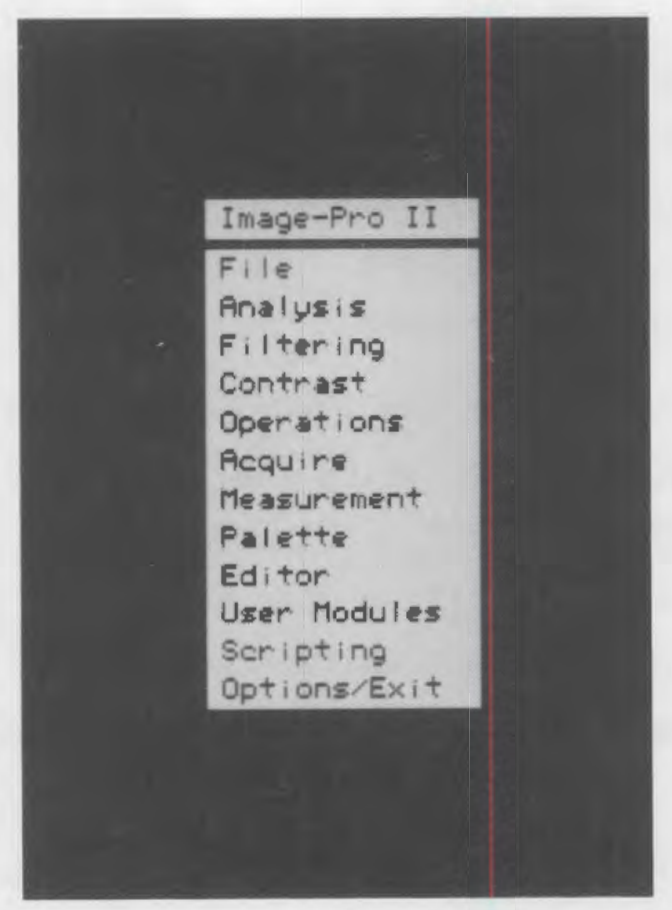

FIGURE 13. The IMAGE-PRO Main Menu 


\section{RADIOMETRIC ANALYSIS PROCEDURES}

The procedure for carrying out radiometric analysis of images is described step by step in this section of the manual. The general flow of steps is as follows: acquire the image to be analyzed; correct a rectangular area of interest for the camera's spatial gain non-uniformity by running the Flatten image user's module; perform a histogram on the area of interest and save the histogram data to disk (this step allows non-rectangular areas of interest to be analyzed); and run the Radiometry user's module, which uses the stored histogram data to calculate the photon flux being emitted by the object.

\section{IMAGE ACQUISITION AND PREPARATION PROCEDURE}

The first set of steps defines the procedure for acquiring an image and preparing the image for quantitative analysis. The following assumes that IMAGE-PRO is running. The specific steps which make up this procedure are:

1. If the image to be analyzed is not currently displayed, load the image from disk, or acquire a frame from live video. Image input and output functions are accessed from the File sub-menu, as shown in Figure 14, and image capture functions are accessed from the Acquire sub-menu, as shown in Figure 15. Although it is possible to place the frame grabber in a real-time average mode via the VS-100 CAPTURE user's module, the software/hardware does not operate properly in this mode, so quantitative analysis of an averaged image is not possible. NOTE: In order to obtain accurate radiometric analyses, the image must be acquired with the microscope's light path selector set to " $\mathrm{C}$ " and the vertical illuminator beamsplitter positioned OUT of the optical path.

2. Make a note of the camera gain setting and the microscope objective used in acquiring the image, as these parameters will be needed at a later stage.

3. Return to the main menu.

4. Save the image to disk. To save the image, first click on the File choice of the main menu. Next click on the Save Image choice of the sub-menu. A list of existing file names and a blank line for a new name appears, as shown in Figure 16. Type the desired file name (up to 8 characters), or click on one of the existing file names (the existing image data will be over-written by the new image). Click on OK when ready to store the image. Click on the Entire Image choice of the Select Area sub-menu, which is shown in Figure 17. The image will be saved. 


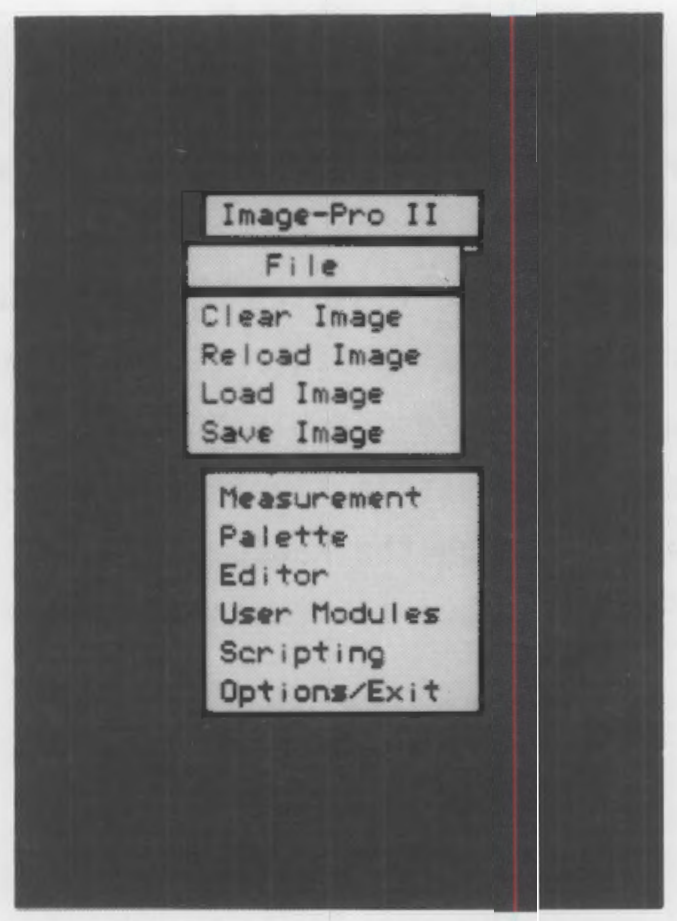

FIGURE 14. The File Sub-Menu. Click on the File Choice of the main menu to display this sub-menu.

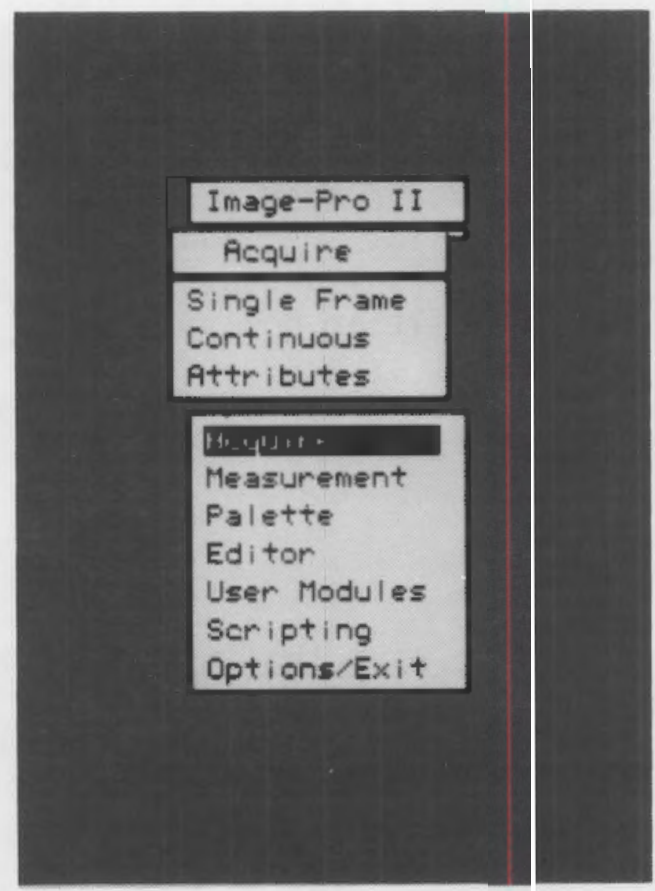

FIGURE 15. The Acquire Sub-Menu. Click on the Acquire choice of the menu to display this sub-menu. 


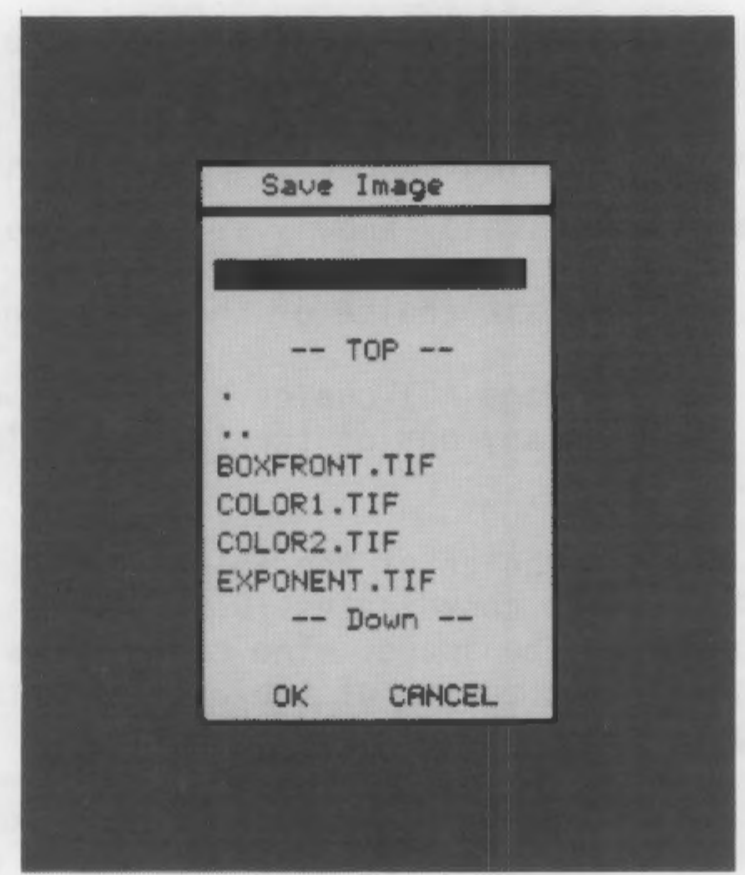

FIGURE 16. The Save Image Directory. Click on the Save Image choice of the File sub-menu to display this directory.

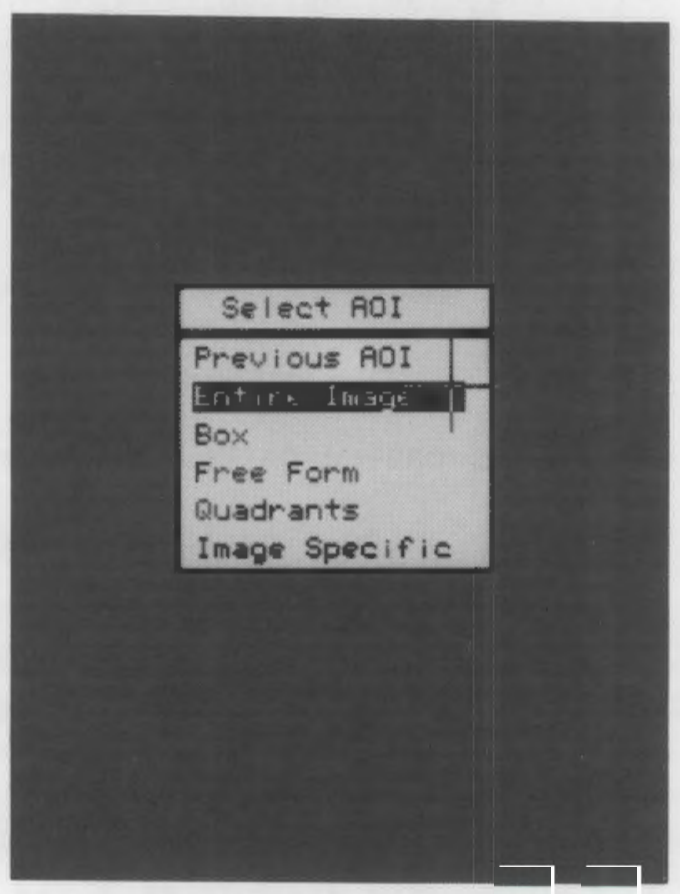

FIGURE 17. The Select Area Sub-Menu. Click on any function which operates on a user-selected area of interest to display this sub-menu. 
Steps 5 through 10 are optional. These steps may be helpful in defining the coordinates of an area of interest (AOI) which will subsequently becorrected for the intensified camera's spatial gain variation. If the necessary coordinates are already known, skip to step 10.

5. Click on the Options/Exit choice of the main menu.

6. Click on the Define Image AOI choice of the sub-menu as shown in Figure 18, and then click on any one of the Image 1 - Image 8 choices, as shown in Figure 19.

7. Click on the New Box choice of the Select AOI sub-menu, as shown in Figure 20. A box with coordinates $(0,0) ;(16,16)$ will appear in the upper left corner of the image. The coordinates of the upper left corner and lower right corner of the area of interest box will appear in the following format:

$$
\begin{aligned}
& \text { (upper left column, upper left row) } \\
& \text { (lower right column, lower right row) }
\end{aligned}
$$

The screen will appear as shown in Figure 21.

8. Adjust the size and location of the box so the box contains the area of interest. The size of the box can be changed by moving the mouse/trackball with the right button held down. The location of the box is controlled by moving the mouse/trackball with no buttons down.

9. When the size and location of the box have been adjusted so that the box contains the area of interest, make a note of the box's two coordinate pairs.

10. Click the left button of the mouse/trackball once to accept the location and size of the box and exit the AOI definition procedure, and then return to the main menu.

11. Click on the User Modules choice on the main menu.

12. Select the Flatten image choice from the User Modules sub-menu, as shown in Figure 22.

13. Messages and prompts from the Flatten image user module appear on the main computer display; so if the video switch box is being used, set the switch to VGA.

The Flatten image module uses the program FLATTEN, which prompts the user for the name of the previously stored image, as well as the coordinates of the area of interest. The program then applies correction factors to adjust for the spatial gain non-uniformity of the camera, and permanently 


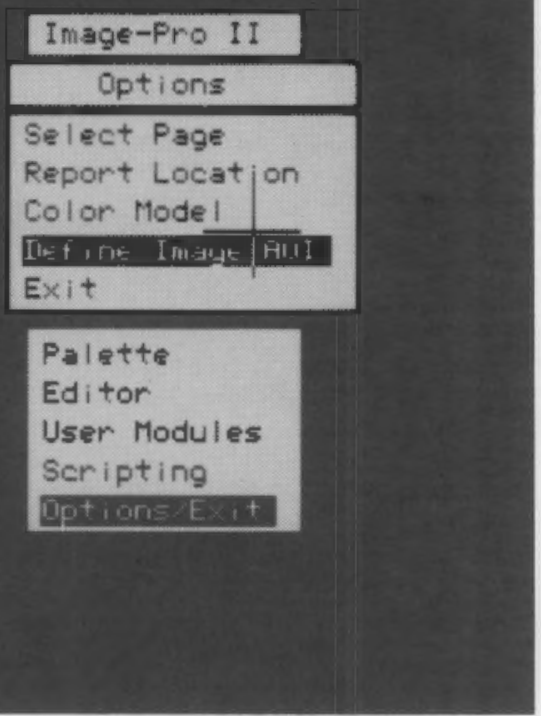

FIGURE 18. The Options Sub-Menu. Click on the Options/Exit Choice of the main menu to display this sub-menu.

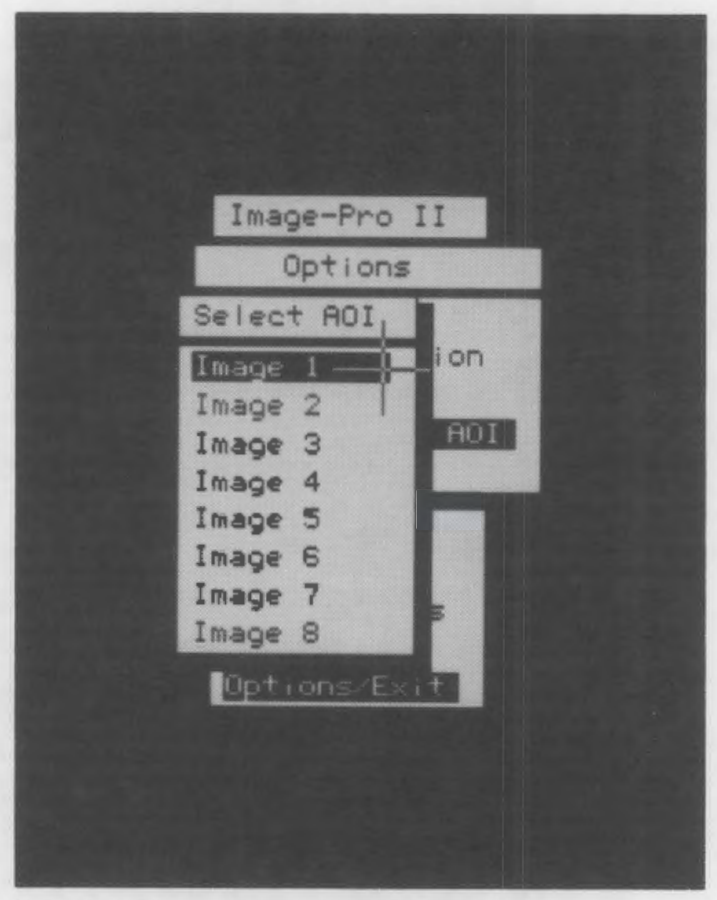

FIGURE 19. The Select AOI Image Sub-Menu. Click on the Define Image AOI choice of the Options sub-menu to display this sub-menu. 


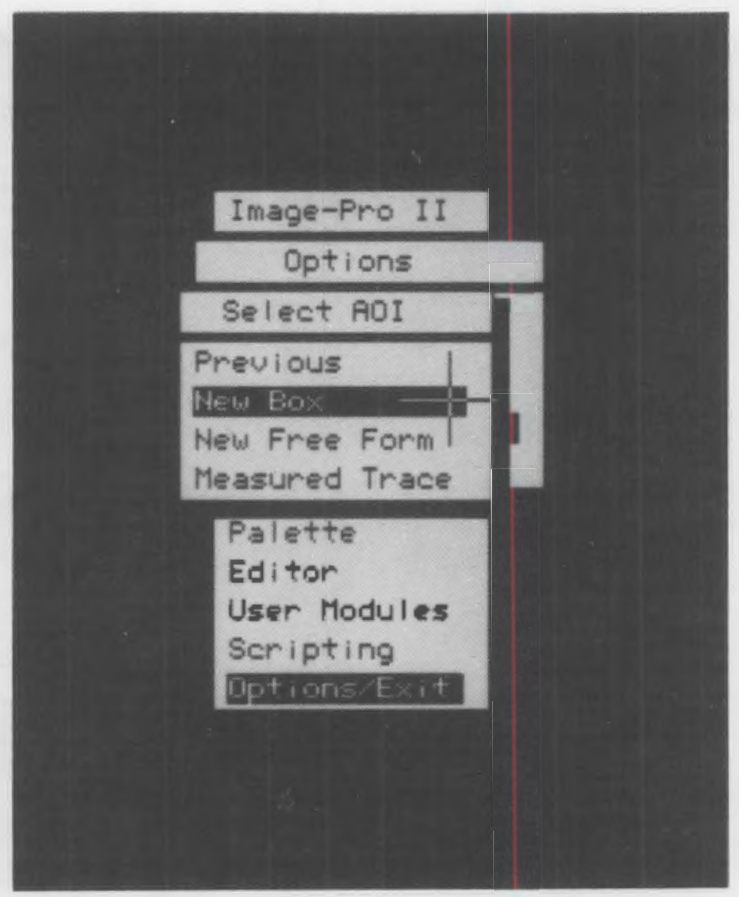

FIGURE 20. The Select AOI Sub-Menu. Click on any of the Image 1 - Image 8 choices of the Define Image AOI sub-menu to display this sub-menu.

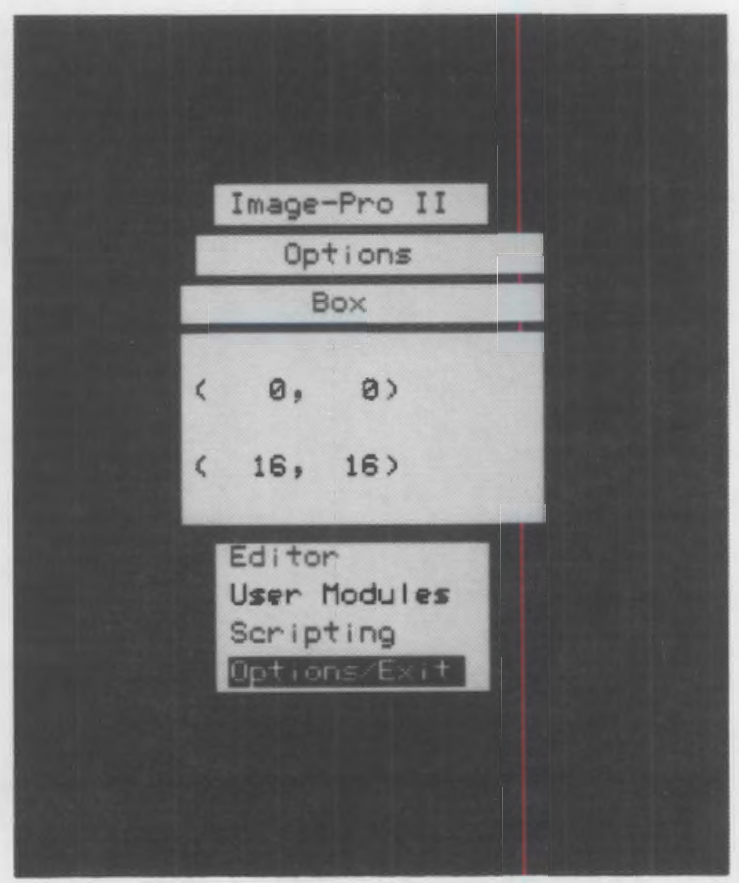

FIGURE 21. The Box Sub-Menu. Click on the New Box choice of the Select AOI sub-menu to display this screen. 


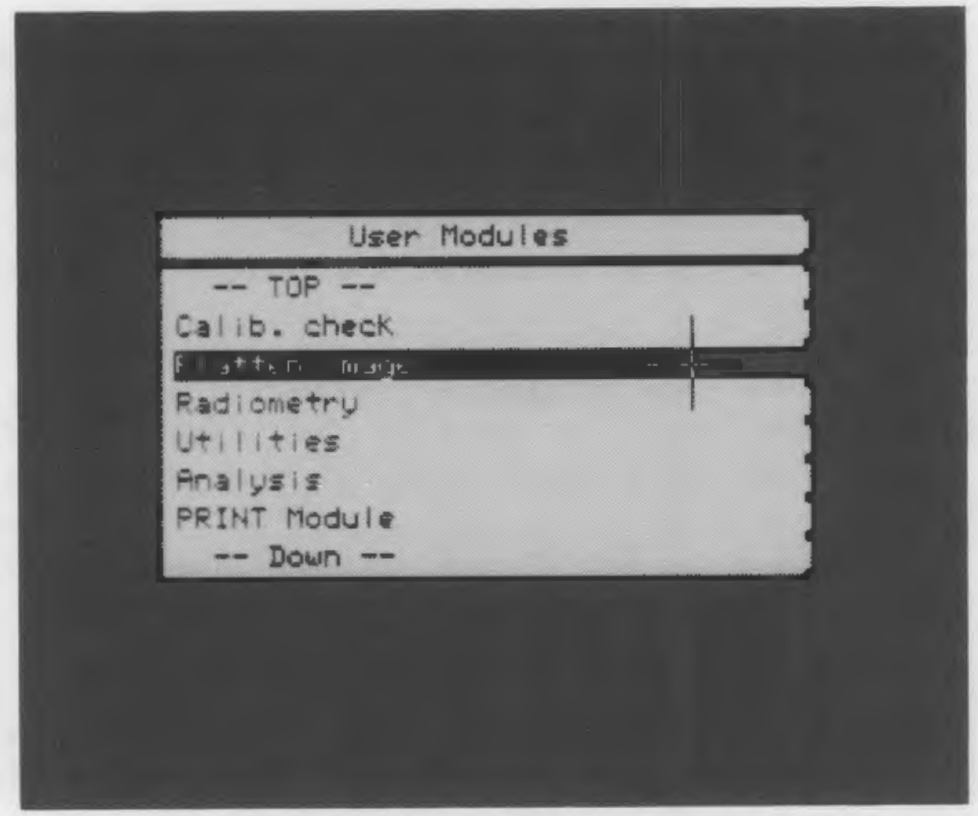

FIGURE 22. The User Modules Sub-Menu. Click on the User Modules choice on the main menu to display this sub-menu.

replaces the old pixels with the new. The newly modified file replaces the original in mass storage. Since the procedure is rather time-consuming, the corrections are only applied to the area of interest chosen by the user, as opposed to the entire image. Note that the user can force FLATTEN to operate on the entire image by entering $(0,0,639,479)$ when prompted for the coordinates of the area of interest. When the Flatten image user module has completed execution, program control will return to the IMAGE-PRO main menu.

14. If the video switch box is being used, set the switch to RGB when the Flatten image module has completed execution.

15. Restore the corrected file from disk by first clicking on the File choice of the main menu. Then click on the Load Image choice of the sub-menu, click on the name of the file that has previously been corrected, and then click on OK to accept the file name.

16. When the corrected image has been restored, radiometric analysis of the image may be performed. 


\section{RADIOMETRIC ANALYSIS PROCEDURE}

With the spatial gain non-uniformity-corrected image displayed, the following steps should be followed to carry out radiometric analysis of the image:

17. Click on the AOI Histogram choice of the Analysis sub-menu, as shown in Figure 23.

18. Click on the desired type of area of interest from the choices on the Select Area sub-menu, and use the mouse/trackball to define the area of interest. Note that the area of interest should lie within the portion of the image that was corrected with the Flatten image user module. Click the left button of the mouse/trackball to accept the area of interest.

19. A histogram of the grey scale content of the image will appear, as well as a listing of the associated statistics. The screen will appear as shown in Figure 24. Click on the OK bar at the top of the statistics block.

20. Answer the Display Accumulated? and Calculate Range of Indexes? prompts, which appear as shown in Figures 25 and 26, as desired. The selection (Yes or No) does not affect the radiometric analysis.

21. The Analysis sub-menu will appear. Click on Save Data. A list of existing data files and a blank line for a new file name will appear, as shown in Figure 27. Enter the name of the data file in which to save the histogram data, and click on OK to initiate the save.

22. Return to the main menu, click on the User Modules choice of the main menu, and click on the Radiometry choice from the sub-menu, as shown in Figure 28 .

23. Messages and prompts from the Radiometry user module appear on the main computer display; so if the video switch box is being used, set the switch to VGA.

The Radiometry user module invokes the program ANALYZE. ANAL.YZE prompts the user for the name of the data file that contains the previously stored histogram data, the camera gain setting, the microscope objective in use, and the name of the file containing the sample spectral content data. The program then calculates and reports the photon flux being generated by the object in the area of interest. Program control will return to the IMAGE-PRO main menu when the Radiometry user module is finished.

24. If the video switch box is being used, set the switch to RGB when the Radiometry module has completed execution. 


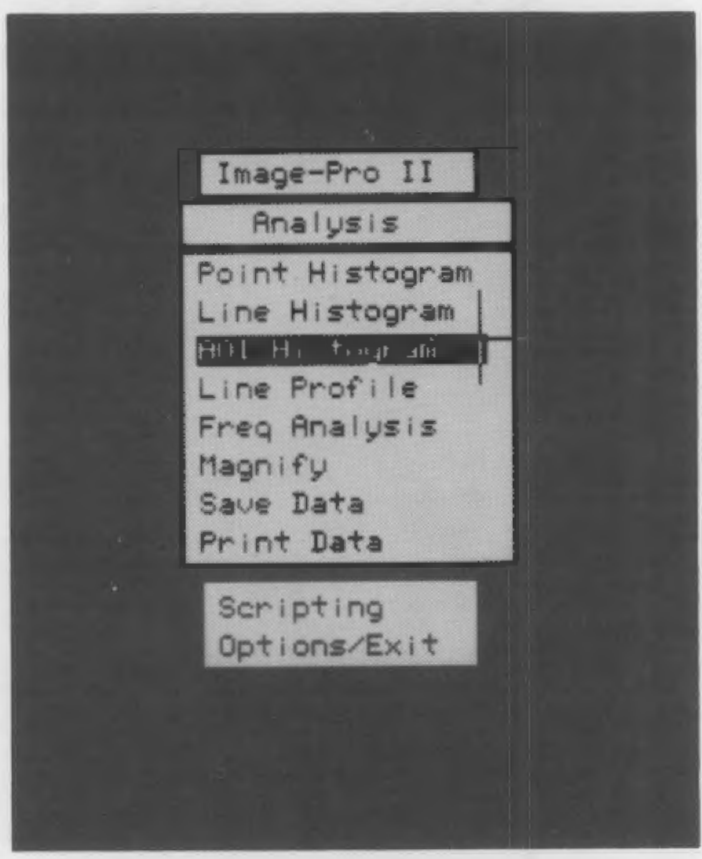

FIGURE 23. The Analysis Sub-Menu. Click on the Analysis choice of the main menu to display this sub-menu.

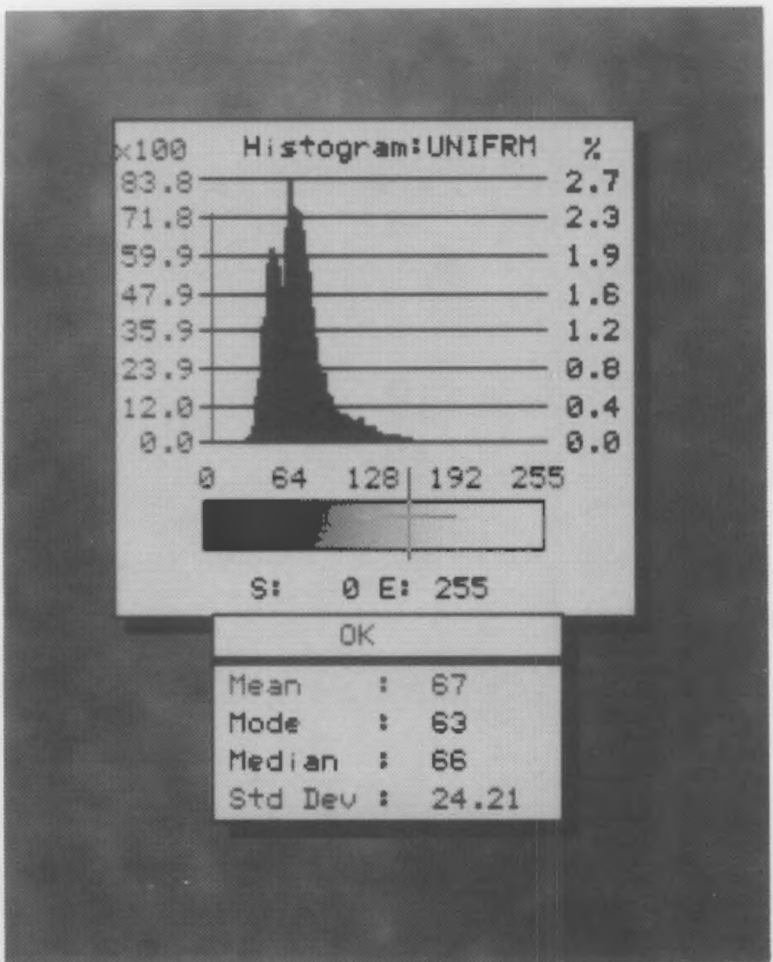

FIGURE 24. The Histogram Screen. Click on the AOI Histogram choice of the Analysis sub-menu, then define the area of interest, to display this screen. 


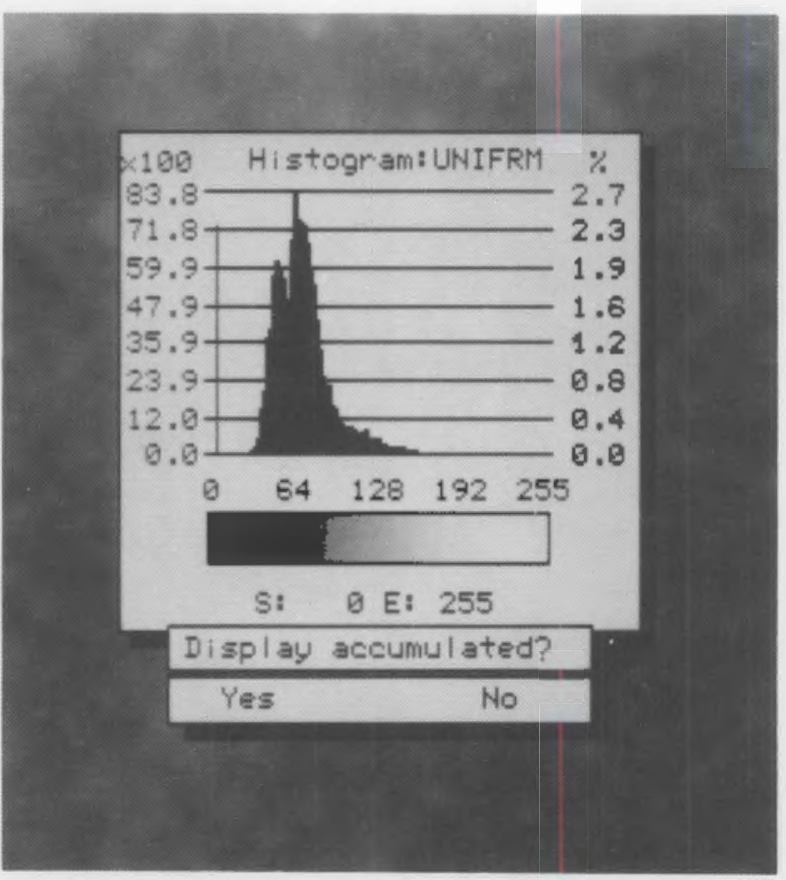

FIGURE 25. The Histogram Screen, Showing the Display accumulated? Prompt

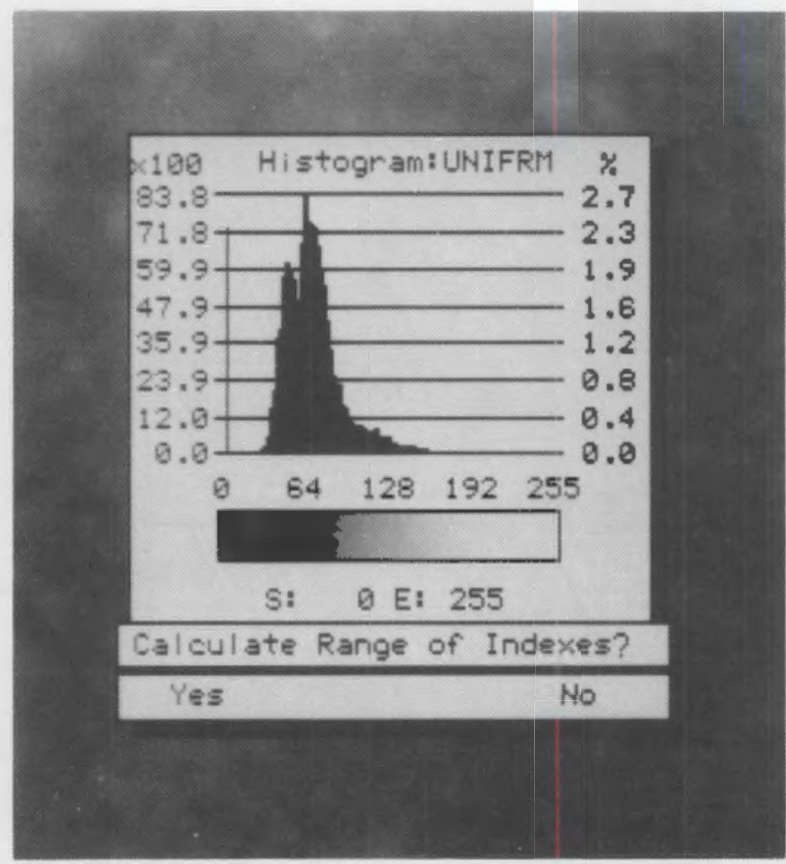

FIGURE 26. The Histogram Screen, Showing the Calculate Range of Indexes? Prompt 


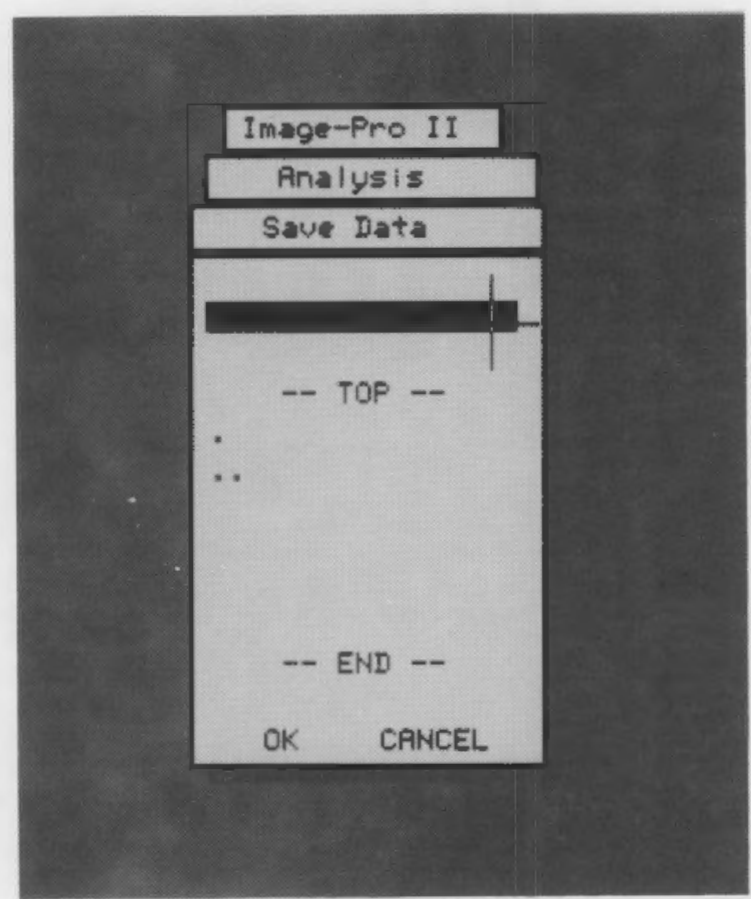

FIGURE 27. The Save Data Directory. Click on the Save Data choice of the Analysis sub-menu, after completing an AOI Histogram, to display this directory.

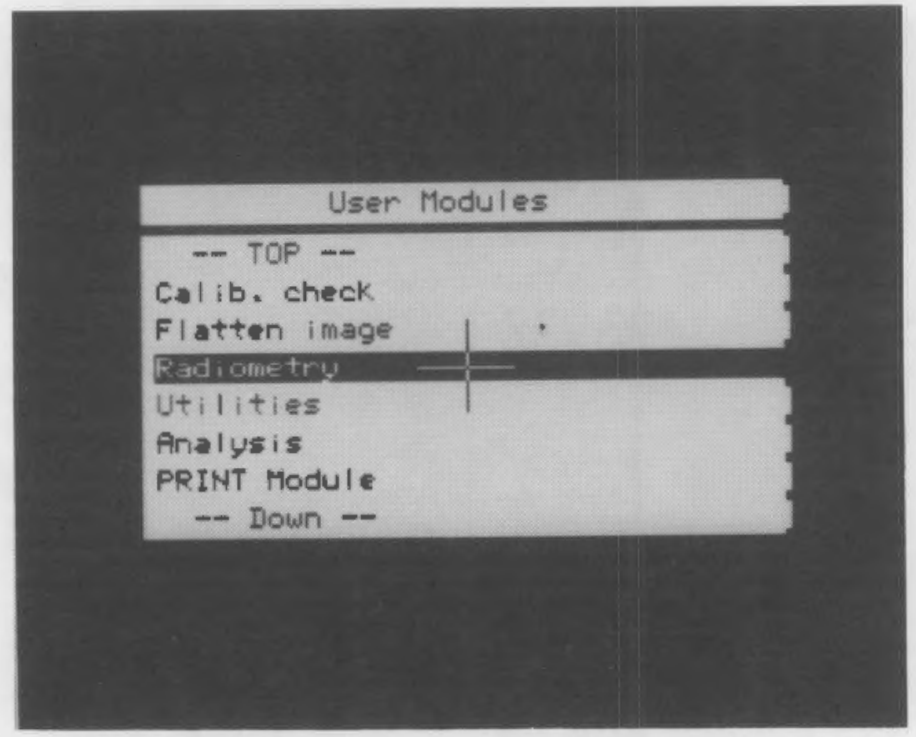

FIGURE 28. The User Modules sub-menu, showing selection of the Radiometry user module. Click on the User Modules choice on the main menu to display this sub-menu. 


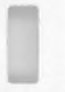

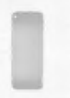

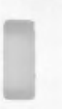

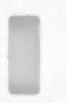

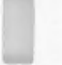

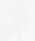

$\square$

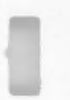

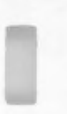

$\square$

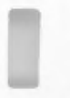

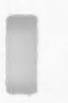

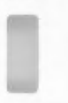

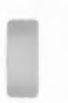

(1)

I

.

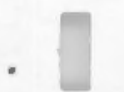

I

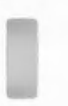

0 


\section{CALIBRATION}

The Radiometry user's module relates the average grey value of an image area of interest to radiometric quantities by using the coefficients of straight lines which are fit to the relationship between grey value, camera gain, and object radiance. This relationship is empirically established during the primary calibration procedure described in the following section. A calibration check procedure which allows slight adjustments in the primary calibration to account for component aging, changing laboratory conditions, etc., is also described below.

\section{PRIMARY CALIBRATION PROCEDURE}

The purpose of the primary calibration procedure is to establish the relationship between the camera gain, average grey value, and calibration source object radiance. The calibration assembly discussed previously in this manual is used to provide a known radiance at the object plane of the microscope. An NIST-traceable radiance source is used to provide a known object radiance. The output of the calibration source is passed through a photometric filter to eliminate spectral content outside the $400 \mathrm{~nm}$ to $700 \mathrm{~nm}$ range, and is focused onto the object plane of the microscope, thereby forming an object of known radiance which can be imaged by the microscope system in the usual manner. The calibration source object spectral radiance, $L_{c o}(\lambda)$ is given by

$$
L_{c o}(\lambda)=L_{c}(\lambda) T_{L}(\lambda) T_{F}(\lambda) R(\lambda)
$$

where $L_{c}(\lambda)$ is the spectral radiance of the NIST-traceable source, $T_{L}(\lambda)$ is the spectral transmittance of the focusing lens, $T_{F}(\lambda)$ is the spectral transmittance of the photometric filter, and $R(\lambda)$ is the spectral reflectance of the mirror used to deflect the converging calibration source radiance up into the microscope's object plane. Note that the magnification of the radiance source at the microscope object plane is approximately unity so that the field of view of the microscope is always overfilled. 
The procedure for characterizing the camera gain, grey value, calibration object source radiance relationship is as follows (the procedure does not need to be carried out on a regular basis, and is presented here for completeness):

1. Adjust the camera gain potentiometer to 0.0 .

2. Adjust the calibration radiance source until the average grey value of the image is nearly 0 .

3. Acquire a frame from continuous video and store the frame to disk.

4. Run the Flatten image user module, and flatten a 200 pixel by 200 pixel box near the center of the image.

5. Load the newly flattened image into the frame grabber memory.

6. Perform a histogram analysis of the 200 pixel by 200 pixel flattened area.

7. Note the average grey value of the flattened area, the camera gain, and percent of full scale reading of the calibration source.

8. Repeat steps 3 through 7 , but adjust the calibration source for increasingly larger average grey values, covering as much of the 0-255 grey value range as possible. Obtain several data points so an analytical function can be fit to the data.

9. Fit a straight line to the data.

10. Complete the above steps for integer settings of the camera gain potentiometer from 0.0 to 10.0 , resulting in a family of straight-line calibration curves.

A typical set of calibration curves is shown in Figure 29. Note that it is not possible to characterize the lowest gain settings for the higher objective powers due to the finite maximum radiance capability of the calibration source. Specifically, gains settings of 0.0 and 1.0 for the $20 x$ objective, and 0.0 for the $10 x$ objective are not characterized.

Note that the entire procedure must be carried out with each of the four microscope objectives in place, since their spectral transmission varies. The data from the calibration is entered in ASCII files which are referenced in the CURVES.CRV file used by the radiometric analysis software. 


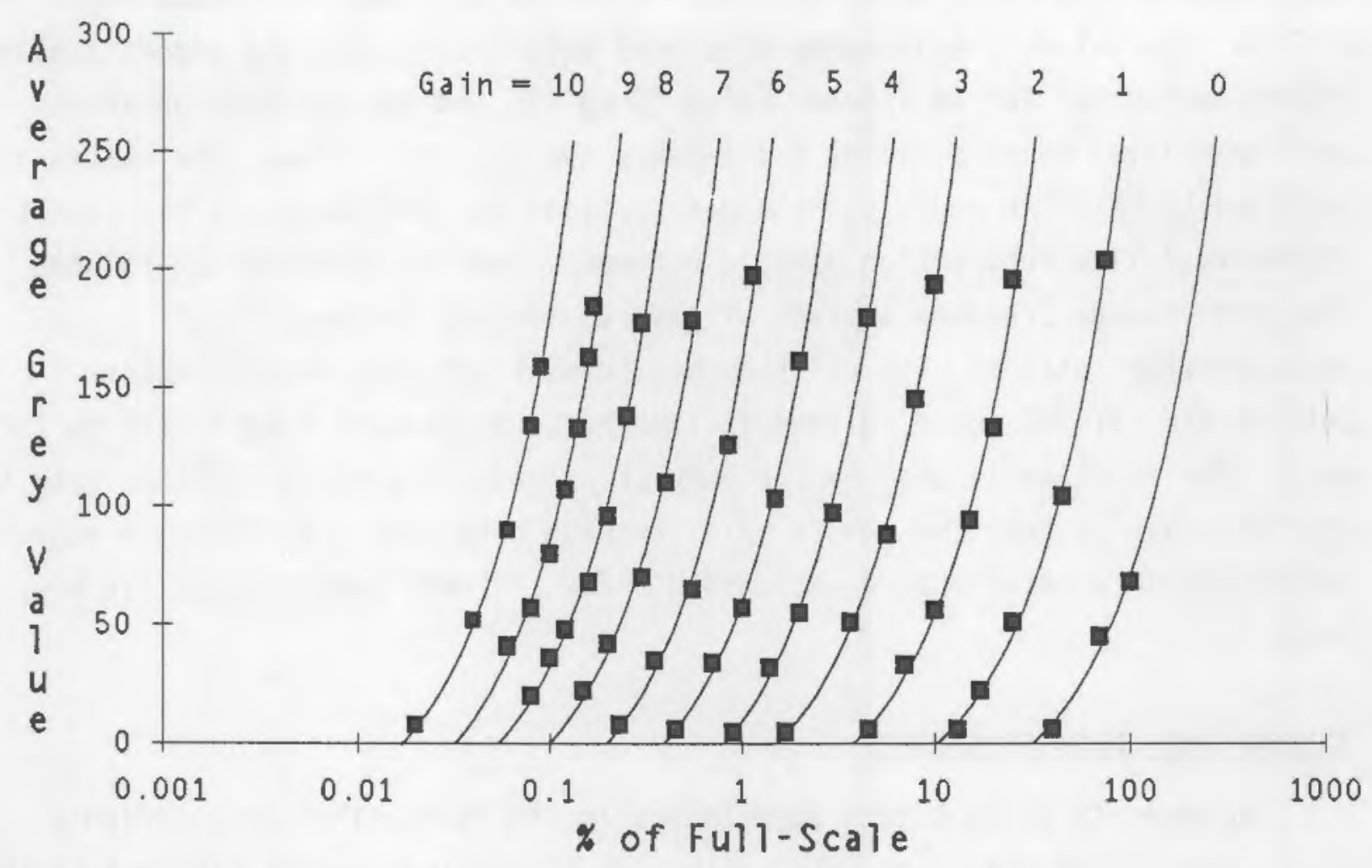

FIGURE 29. Primary Calibration Curves for the 2.5x Objective

An additional consideration is the fact that the grey value distribution in a given image becomes wider as the gain increases. Therefore, at the higher gains, it may not be possible to reach the very low and very high average grey values without having a large fraction of the pixels registering either 0 or 255; that is, the average grey value is skewed by the low- or high-saturated pixels. These data points are not used in fitting a line to the data; their un-skewed values are extrapolated from the data for the more well-behaved grey value ranges.

The relationship between camera gain, grey value, and calibration object source radiance, for the case of EXTERNAL gain operation of the camera, is derived from the relationship for INTERNAL gain control operation. The realtime image subtraction capability of the frame grabber is used to find the 
EXTERNAL gain voltage that gives an amount of gain equal to a given gain potentiometer (INTERNAL mode) setting. A reference frame (the frame which will be subtracted from incoming video) is established with the camera's gain potentiometer set for an integer value $(0$ to 10$)$ and the calibration source set for a level which provides a mid-range average grey value. The camera is switched to EXTERNAL mode, and the gain voltage is adjusted until the result of the real-time subtraction gives a minimum value; the minimum occurs when the gain voltage produces a level of gain equivalent to the INTERNAL potentiometer setting. The relationship between INTERNAL potentiometer setting and EXTERNAL gain voltage is linear, so a straight line is fit to the data. The equation is used by the ANALYZE program to convert EXTERNAL gain to INTERNAL gain so that the camera gain, average grey value, calibration source object radiance relationship determined under INTERNAL gain control can be used.

\section{CALIBRATION CHECK PROCEDURE}

In order to allow slight adjustments in the calibration on a periodic basis, the system can be quickly calibrated by placing a known radiance at the object $\mathrm{plane}$ of the microscope and using the resulting average grey value of the image to find the "expected" percent of full-scale setting of the calibration source, based on the primary calibration data. The results of the subsequent radiometric calculations are then multiplied by the ratio of the expected percent of full-scale setting to the actual setting (this ratio will be referred to as the calibration factor). If this ratio is greater than one, this is an indication that the system has become slightly less sensitive than it was at the time of the primary calibration, and the calculated radiometric quantities are scaled up by the proper factor. If the ratio is less than one, the system has become more sensitive, and the quantities are scaled down by the proper factor.

It is recommended that the calibration be carried out frequently. A realistic interval between calibrations should become clear as the user gains experience with the M-QLIS; however, if the operating environment of the system changes significantly (system is moved to a different laboratory, 
etc.), the calibration should certainly be carried out before undertaking quantitative analysis.

Experience with the intensified camera has shown that the camera may require quite a long period of time to establish a stable level of gain when a large change in illumination level is encountered. Therefore, as the calibration check procedure shows, it is recommended that the calibration check be carried out with the calibration source set for a level which gives an average grey value that is similar to the grey values expected from the specimen.

The steps for completing the calibration check procedure are as follows:

1. Turn the intensified camera on and allow approximately 1/2-hour warm-up time.

2. Set up the calibration source system as described in the ASSEMBLY AND OPERATION portion of this manual, and allow at least 5 minutes of warmup time for the calibration source.

3. Start the IMAGE-PRO software as outlined in the Software section of this manual, and select the Continuous capture mode from the Acquire submenu, so that the video output of the intensified camera can be monitored continuously.

4. After focusing the camera on the coincident reticle and cross-hair pattern, slide the microscope light path selector to "V" to prevent over exposure of the camera during subsequent adjustments.

5. Set the camera gain to 0 .

6. Remove both the reticle and the cross-hair insert, and place the aperture/filter holder, with the $3 / 4$ " aperture and the photometric filter, in the calibration source output port.

7. Slide the microscope light path selector to " $C$ " to send the image up to the camera.

8. If the vertical illuminator beamsplitter is in the optical path, slide the beamsplitter out of the optical path.

9. Rotate the desired objective into position.

10. Set the camera gain and the calibration source level so the average grey value of the resulting image is similar to the grey values expected from the specimen. 
11. Acquire an image from the continuous video by clicking the left button of the mouse/trackball.

12. Click on the AOI Histogram choice of the Analysis sub-menu.

13. Click on the Entire Image choice on the Select Area sub-menu. A histogram of the grey scale content of the image will appear, as well as a listing of the associated statistics. Note- the mean value shown in the statistics box.

14. Answer the Display Accumulated? and Calculate Range of Indexes? prompts as desired.

15. Repeat steps 10 through 13 at 1 - or 2 -minute intervals until the mean grey value has stabilized.

16. Note the camera gain, the objective in use, and the percent of fullscale setting of the calibration source; these parameters are required when the Calib. check user module is run.

17. Return to the main menu.

18. Save the image to disk. To save the image, first click on the File choice of the main menu. Next click on the Save Image choice of the sub-menu. A list of existing file names and a blank line for a new name appears. Click on the existing file named CALIMAGE (the existing image data will be over-written by the new image). Click on OK when ready to store the image. Click on the Entire Image choice of the Select Area sub-menu. The image will be saved.

19. Click on the User Modules choice on the main menu.

20. Select the Calib. check choice from the User Modules sub-menu.

21. Messages and prompts from the Calib. check user module appear on the main computer display, so if the video switch box is being used, set the switch to VGA.

The Calib. check module uses the program CALCHECK, which prompts the user for the camera gain setting, the objective in use, and the percent of full-scale setting of the calibration source. The program then opens the image file (CALIMAGE.TIF) and applies correction factors to adjust for the spatial gain non-uniformity of the camera over a 200 pixel by 200 pixel area in the central portion of the image, and finds the average grey value in this area. The program then finds the "expected" percent of full-scale calibration source setting, based on the primary calibration curves discussed in the Primary Calibration Procedure section of this manual. The expected value and 
the actual value are stored in the CURVES.CRV file, and are used as previously described. When the Calib. check user module has completed execution, program control returns to the IMAGE-PRO main menu.

22. If the video switch box is being used, set the switch to RGB when the Calib. check module has completed execution. 



\section{RADIANCE CALCULATIONS}

The radiometric calculations performed by the Radiometry user's module convert video grey scale levels to the corresponding number of photon flux (in units of photons/second) being generated by the chemiluminescent sample. In general terms, the calculations proceed as follows: the image to be analyzed is corrected for the camera's spatial gain non-uniformities; the average grey value of the area of interest in the image is found; using the average grey value, the camera gain setting, and the microscope objective identification, the corresponding calibration source radiance is found from the primary calibration curves; this calibration radiance is multiplied by the previously determined calibration factor to adjust for slight variations in system sensitivities; the difference in the M-QLIS response to the spectral content of the sample and the calibration source are taken into account; the adjusted calibration radiance is converted to total flux (power); the sample spectral content is divided into wavelength bands, and the number of photons contributing to the total flux is found for each band; and the number of photons from each band is summed, giving the total photon flux being generated in the image area of interest.

The ANALYZE program first calculates the average grey value, $v_{a v g}$, of the area of interest, and then determines the calibration source radiance that gives an equivalent grey value. Due to the adjustable gain of the intensified camera, any particular grey value can correspond to many different radiance levels. In order to assign the proper equivalent calibration source object radiance $L_{c o}$ to the grey scale value, a set of analytical functions (the previously discussed primary calibration curves) which describe the relationship between grey value, camera gain, and percent of full-scale calibration source radiance are used. Given a particular camera gain setting, microscope objective, and grey value, the equivalent $L_{c o}$ value is found by multiplying the $100 \%$ of full-scale total radiance value by the percent of full-scale value from the appropriate primary calibration curve. The ANALYZE program asks the user to identify the objective in use, camera gain setting, and sample spectral content. 
The sample spectral content is important because it will, in general, differ somewhat from the calibration source spectral content. Since the microscope optics and the intensified camera have wavelength-dependent characteristics, the radiance calculation must account for the unequal efficiency with which the light from the sample propagates through the optical system and is detected by the camera, as compared to that for light from the calibration source. This variability can be accounted for by considering ratio of the integral of the spectral content of the calibration source multiplied by the spectral response of the detection system (optics and camera) to the integral of the spectral content of the sample multiplied by the spectral response of the detection system. Since the intensified camera is a spectrally integrating device (that is, the spectral content of the object is lost at the photocathode of the image intensifier), taking the ratio of these integrals is a valid method of accounting for the different spectral qualities of the sample and the calibration source. In equation form, this ratio is $\xi_{c} / \xi_{s}$ where

$$
\xi_{c}=\int_{\lambda_{1}}^{\lambda_{2}} M(\lambda) T_{0 b j}(\lambda) C(\lambda) d \lambda
$$

and

$$
\xi_{S}=\int_{\lambda_{1}}^{\lambda_{2}} M(\lambda) T_{\text {obj }}(\lambda) S(\lambda) d \lambda
$$

and $M(\lambda)$ is the spectral response of the microscope body and intensified camera, $\operatorname{Tobj}_{o j}(\lambda)$ is the spectral transmittance of the microscope optics (which varies according to which objective is in use), $C(\lambda)$ and $S(\lambda)$ are the calibration source and sample spectral contents, respectively, and $\lambda$ is the wavelength. Since both the calibration source and typical sample spectra have very little content outside the wavelength range of $400 \mathrm{~nm}$ to $700 \mathrm{~nm}$, these limits are used as the limits of integration in the ANALYZE program.

A periodic calibration check provides a means of accounting for slight changes in the response of the system due to temperature dependencies in the electronics, aging of components, etc. The calibration check consists of placing a known radiance at the microscope's object plane, and using the 
resulting average grey value of the image to find the "expected" percent of full-scale setting of the calibration source, based on the primary calibration data. The calculated radiance of the sample is multiplied by the ratio of the expected percent of full-scale setting $s_{e}$ to the calibration check percent of full-scale setting $s_{C}$.

The ANALYZE program calculates the radiance $L$ of the object by combining the factors described above. In equation form,

$$
L=\frac{s_{e}}{s_{c}} \frac{\xi_{c}}{\xi_{s}} L_{c o}
$$

Note that $L$ has units of watts $/ \mathrm{cm}^{2}-s r$.

After the radiance has been determined, the ANALYZE program calculates the photon flux being emitted by the sample. The program first accounts for the angular dependance of the radiance by calculating the radiant exitance (the flux per unit area leaving the surface of the sample), which is simply $\pi \mathrm{L}$ for a Lambertian source (a Lambertian source is one whose radiance is independent of viewing angle, a description which is fitting for the chemiluminescence being considered).

The radiant flux (or power) $P$, in units of watts, is calculated by multiplying the radiant exitance by the actual area, in $\mathrm{cm}^{2}$, of the area of interest. The area per pixel for each microscope objective was found by imaging targets of known dimensions and using the image processor to find the corresponding number of pixels making up the target image. The ANALYZE program finds the area contained in the area of interest by simply counting the number of pixels in the area of interest and multiplying by the $\mathrm{cm}^{2} / \mathrm{pixel}$ value appropriate for the objective in use.

After calculating the power, the ANALYZE program calculates the photon flux (photons/second) being emitted by the sample. The calculation begins with an integration of the sample spectral content. Following this, the spectrum is divided into discrete bands $5 \mathrm{~nm}$ wide. The integral of the each band is divided by the integral of the entire curve so that the fraction $F(\lambda)$ of the total spectrum contained in each band is known, and hence the power contributed by each band. Next, the energy of a photon with the wavelength 
lying in the center of each band is calculated in eV per photon and is subsequently converted to joules/photon. Since 1 joule $=1$ watt-second, this energy per photon, $E_{p}$, can be expressed in watt-seconds per photon. Thus, the photon flux $\mathrm{N}_{\mathrm{ph}}(\lambda)$, in photons per second, in each band is found by

$$
N_{p h}(\lambda)=\frac{P F(\lambda)}{E_{p}(\lambda)}
$$

The total photon flux is calculated as the sum of the $\mathrm{N}_{\mathrm{ph}}$ 's for each band. 


\section{APPENDIX A}

\section{COMPONENT VENDOR LIST}


APPENDIX A

\section{COMPONENT VENDOR LIST}

Microscope

Model \#BHMJ

Olympus Corporation

Precision Instrument Division

1 Centerpointe Drive

Suite 430

La Palma CA 90623

714-670-1324

\section{Ca]ibration Source}

Model \#RS-65-C

Hoffman Engineering Corporation

20 Acosta Street

Stamford CT 06902

203-325-8933

\section{Intensified Camera}

Model \#F4577

ITT Electro-Optical Products Div.

3700 East Pontiac Street

P.0. Box 3700

Fort Wayne IN 46801

219-423-4341

\section{Computer}

Model \#38620

Gateway 2000

P.0. Box 2000

Sergeant Bluff IA 51054

800-523-2000
Multi-sync Monitor

Mode1 \#JC1404HMA1

NEC Home Electronics (USA), Inc. 1255 Michael Drive Wood Dale IL 60191

312-860-9500

Optical Disk Drive

Model \#PC-INSPIRE

Alphatronix, Inc. 2300-T Englert Drive, Suite C Durham NC 27713

919-544-0001

Frame Grabber/Processor

Mode1 \#VS-100/640

Imaging Technology, Inc. 6000 West Cummings Park Woburn MA 01801

617-938-8444

Image Processing Software

Model \#Image-Pro II

Media Cybernetics

8484 Georgia Avenue

Silver Spring MD 20910

800-446-4256

Fixed Voltage Power Supply

Model \#LM15.6-L

AAK Corporation

747 River Street

Haverhill MA 01832

508-373-3769 
Variable Voltage Power Supply

Model \#1635

BK Precision

Maxtec International Corporation

6470 West Cortland Street

Chicago IL 60635

312-889-1448

Sample Dishes for RF Sample Holder

Model \#25382 (Corning 25000)

VWR Scientific

P.0. Box 3551

Seattle WA 98124

206-575-1500 
APPENDIX B

RF SAMPLE HOLDER DETAILS 
The RF sample holder provides a means of coupling RF radiation to a test sample, without precluding the use of a standard microscope to view the resulting reactions. The sample holder is essentially a section of $50 \mathrm{ohm}$ coaxial waveguide which is terminated in a cavity which is designed to maximize power transfer to a sample of water. The sample holder is large enough to hold a $35 \mathrm{~mm}$ diameter culture dish at the waveguide's output end. The center conductor of the sample holder is slightly recessed so that the culture dish is enclosed by the outer conductor. Dimensioned drawings of the sample holder are presented in Figure B.1 and B.2.

\section{ALL UNITS ARE IN INCHES}

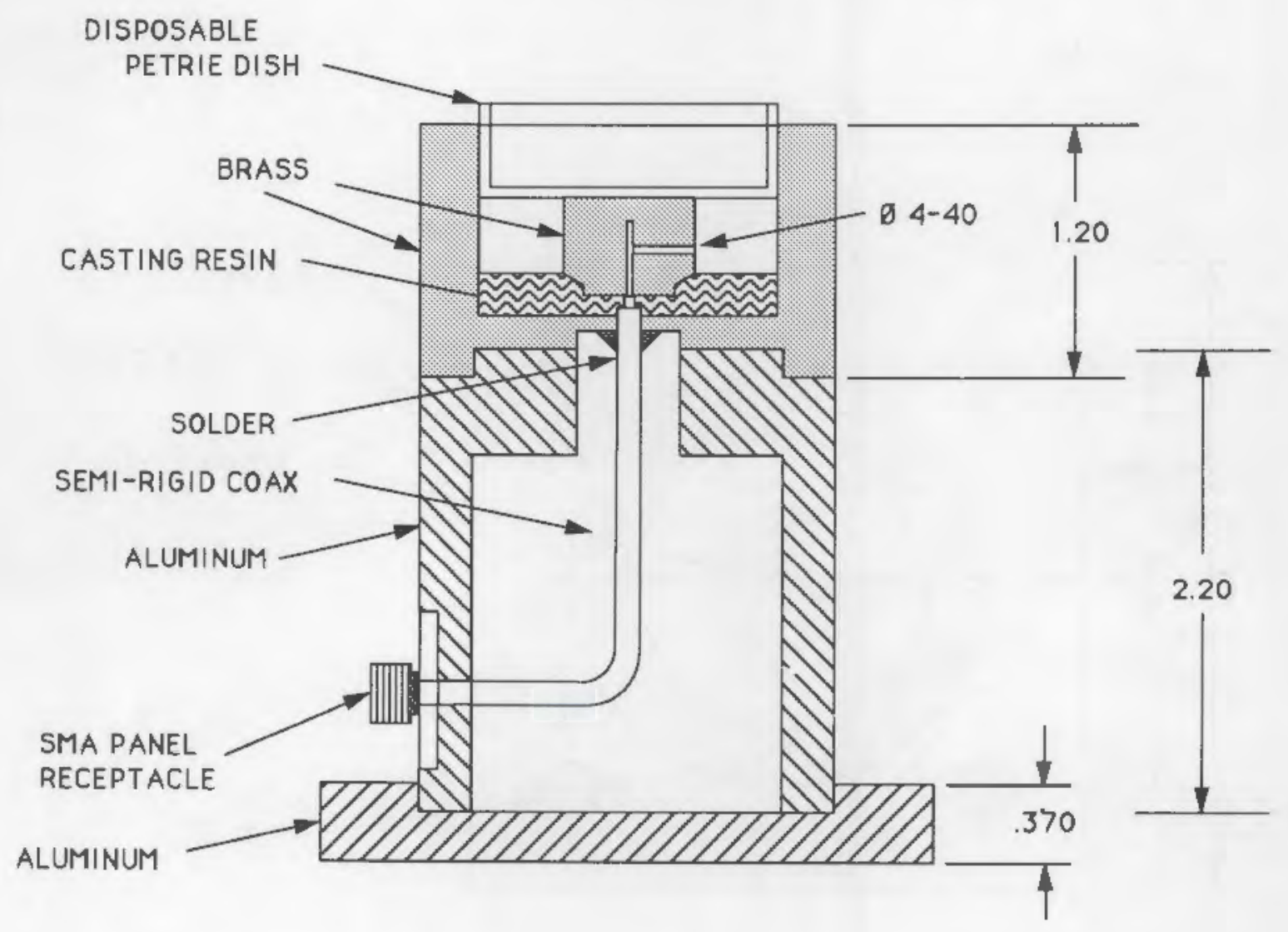

FIGURE B.1. RF Sampler Holder, Elevation View 
ALL UNITS ARE IN INCHES

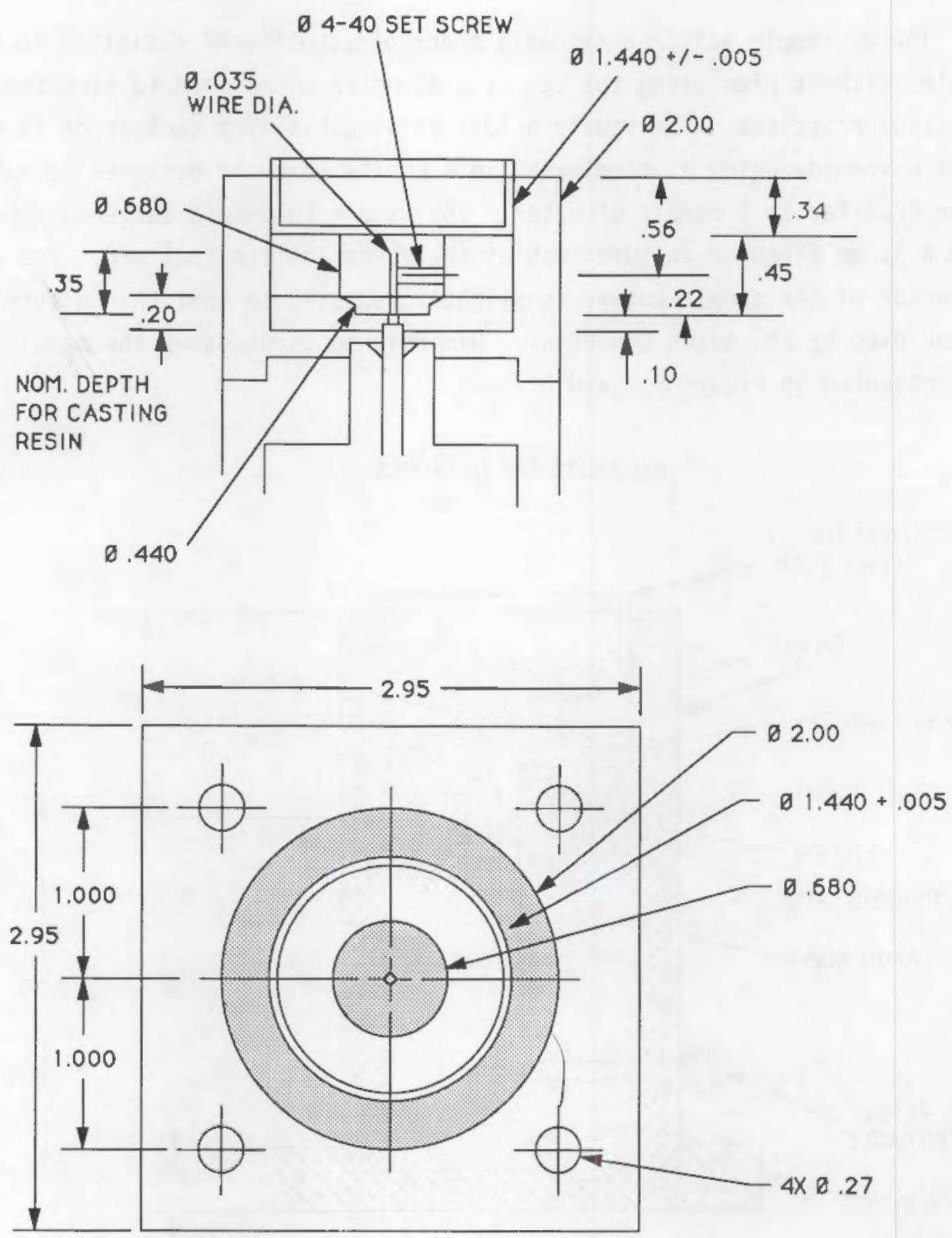

FIGURE B.2. RF Sample Holder, Elevation Detail (Top); Plan Detail (Bottom)

\section{B.2}


The design frequency for the sample holder was $2.45 \mathrm{GHz}$. This frequency was chosen since it is commonly used in commercial microwave oven applications and should, therefore, be useful for the investigations involving the M-QLIS. An additional benefit is the good availability of power supplies which operate at this frequency.

The RF sample holder represents a significant improvement in power delivered to the sample and in uniformity of the field, in comparison to the previous design. A plot of microwave power distribution for the new and old RF sample holders is given in Figure B.3. An un-calibrated vertical monopole probe antenna mounted to a micrometer-driven translation stage was used to sample the field at several points along a radius from the center of a sample dish placed in each holder. The sample dish contained 0.12 in. of water. The measurement conditions for each sample holder were nominally the same; however, neither sample holder has been thoroughly characterized, so the results presented here must be regarded as preliminary. The preliminary data in Figure B.3 indicates that a) the new RF sample holder delivers over two orders of magnitude more power than the previous design and $b$ ) the uniformity of the new design is improved by a factor of approximately five with respect to that of the previous design. 


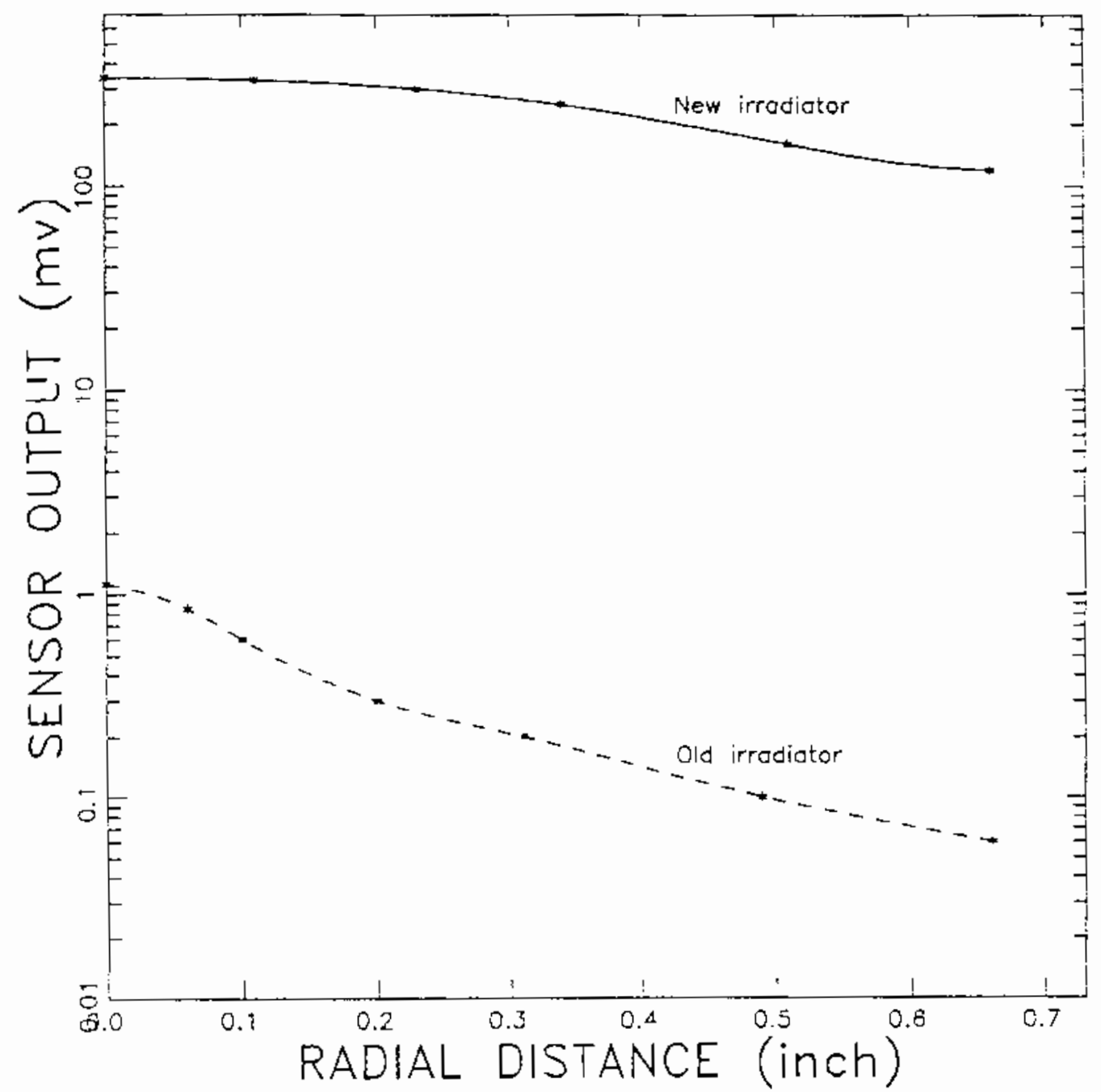

FIGURE B.3. Radiated Microwave Power Distribution for New and 01d RF Sample Holder 


\section{DISTRIBUTION}

No. of

Copies

\section{OFFSITE}

6 J. L. Kiel

Directed Energy Division

Armstrong Laboratory/OEDR

Brooks Air Force Base TX 78235

2 DOE/Office of Scientific and

Technical Information

\section{ONSITE}

DOE Field office, Richland

J. J. Sutey

9 Pacific Northwest Laboratory

C. R. Batishko

K. A. Stahl

Intellectual Property Services

Publishing Coordination

Technical Report Files (5) 
